\title{
The ChvG-ChvI and NtrY-NtrX two-component systems coordinately regulate growth of Caulobacter crescentus
}

\author{
Benjamin J. Stein, Aretha Fiebig, Sean Crosson ${ }^{\#}$ \\ Department of Microbiology and Molecular Genetics, Michigan State University, East Lansing, \\ MI, USA \\ \#Correspondence: crosson4@msu.edu
}

Running Title: Coordinated regulation by two conserved signaling systems

\begin{abstract}
Two-component signaling systems (TCSs) are comprised of a sensory histidine kinase and a response regulator protein. In response to environmental changes, sensor kinases directly phosphorylate their cognate response regulator to affect gene expression. Bacteria typically express multiple TCSs that are insulated from one another and regulate distinct physiological processes. There are certainly examples of cross-regulation between TCSs, but this phenomenon remains relatively unexplored. We have identified regulatory links between the ChvG-ChvI (ChvGI) and NtrY-NtrX (NtrYX) TCSs, which control important and often overlapping processes in $\alpha$-proteobacteria, including maintenance of the cell envelope. Deletion of $\operatorname{ch} v G$ and $\operatorname{ch} v I$ in Caulobacter crescentus limited growth in defined medium and a selection for genetic suppressors

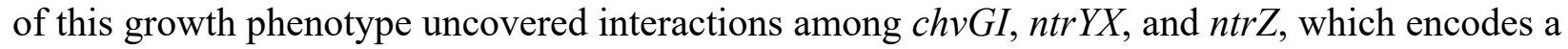
previously uncharacterized periplasmic protein. Significant overlap in the experimentally-defined ChvI and NtrX transcriptional regulons provided support for the observed genetic connections

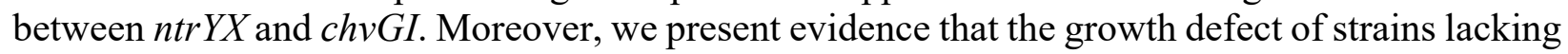
chvGI is influenced by the phosphorylation state of NtrX and, to some extent, by levels of the TonB-dependent receptor ChvT. Measurements of NtrX phosphorylation in vivo indicated that $\mathrm{NtrZ}$ is an upstream regulator of NtrY, and that NtrY primarily functions as an NtrX phosphatase. We propose a model in which NtrZ functions in the periplasm to inhibit NtrY phosphatase activity; regulation of phosphorylated NtrX levels by NtrZ and NtrY provides a mechanism to modulate and balance expression of the NtrX and ChvI regulons under different growth conditions.
\end{abstract}

\section{Importance}

Two-component signaling systems (TCSs) enable bacteria to regulate gene expression in response to physiochemical changes in their environment. The ChvGI and NtrYX TCSs regulate diverse pathways associated with pathogenesis, growth, and cell envelope function in many $\alpha$ proteobacteria. We used Caulobacter crescentus as a model to investigate regulatory connections between ChvGI and NtrYX. Our work defined the ChvI transcriptional regulon in C. crescentus and revealed a genetic interaction between ChvGI and NtrYX, whereby modulation of NtrYX signaling affects the survival of cells lacking ChvGI. In addition, we identified NtrZ as a periplasmic inhibitor of NtrY phosphatase activity in vivo. Our work establishes C. crescentus as an excellent model to investigate multi-level regulatory connections between ChvGI and NtrYX in $\alpha$-proteobacteria. 


\section{Introduction}

Bacteria employ two-component signaling systems (TCSs) to respond to environmental cues and maintain cellular homeostasis (1). TCS sensory modules consist of two core components: a sensory histidine kinase (HK) and a response regulator (RR). In response to signal(s), the HK undergoes autophosphorylation on a conserved histidine residue, and then passes the phosphoryl group to a conserved aspartate on the receiver (REC) domain of the RR (1). HKs may also act as phosphatases, dephosphorylating phosphorylated RRs (1). RR phosphorylation generally alters the activity of effector domains that change gene expression. TCSs are modular, and the output of a particular RR may vary between different organisms (2-5). TCSs that regulate host interactions in pathogens and symbionts are often conserved in related free-living organisms and enable responses to similar physiochemical cues present in the environment $(2,6-8)$.

Although typical TCSs rely on a single HK and RR pair, many systems incorporate additional proteins, such as activators or inhibitors, to form more complex signaling networks (9-17). Historically, most TCSs have been considered to be insular systems, but in many bacteria, crossregulation between HKs and RRs may integrate multiple environmental cues (9, 11, 14, 18-20). Even when HK and RR pairs are well insulated, TCSs can interact at the transcriptional level (20, 21). For example, one TCS may regulate the expression of other TCS genes, or multiple TCSs may influence transcription of the same downstream gene (22-24).

ChvG-ChvI (ChvGI) and NtrY-NtrX (NtrYX) are conserved $\alpha$-proteobacterial TCSs that often regulate similar physiological processes, raising the possibility that they may work together in a coordinated fashion $(25,26)$. The ChvG HK and ChvI RR were originally identified as pleiotropic regulators in the plant pathogen Agrobacterium tumefaciens, affecting virulence, detergent tolerance, and $\mathrm{pH}$ sensitivity $(8,27)$. Subsequent work has linked ChvGI to host interaction, cell motility, acid sensing, and exopolysaccharide production in a variety of $\alpha$-proteobacteria $(6,7,28$ 32). In most characterized systems, the periplasmic protein ExoR binds to ChvG and inhibits its kinase activity $(15,16,33)$. Acidic $\mathrm{pH}$ activates the ChvGI system by triggering rapid proteolysis of ExoR (34). However, not all organisms with ChvGI, including Caulobacter crescentus, encode an ortholog of ExoR. These bacteria must regulate ChvG kinase activity by a different mechanism.

Like ChvGI, NtrYX (consisting of the NtrY HK and NtrX RR) is conserved in many $\alpha$ proteobacteria, including multiple pathogens and symbionts (35-39). Although early studies concluded that NtrYX regulates nitrogen metabolism, recent work suggests that, in certain $\alpha$ proteobacteria, it also controls exopolysaccharide biosynthesis, cell motility, and cell envelope composition $(25,35,39,40-44)$. Given that these processes are also regulated by ChvGI, it is conceivable that ChvGI and NtrYX act coordinately. However, no work to date has identified a substantial genetic interaction between these TCSs $(25,26)$.

C. crescentus, a free-living $\alpha$-proteobacterium found in freshwater and soil environments, encodes both the ChvGI and NtrYX systems $(45,46)$. C. crescentus ChvGI activates transcription of the small regulatory RNA $c h v R$, which post-transcriptionally represses the TonB-dependent receptor gene $\operatorname{ch} v T$ (6). Examination of reporters of $c h v R$ transcription indicated that ChvGI is activated by growth in defined medium, acidic $\mathrm{pH}$, DNA damage, growth at stationary phase, and cell envelope stress $(6,47)$. However, aside from $c h v R$, genes regulated by ChvGI have not been defined. $C$. crescentus NtrYX is less well-characterized than ChvGI, but a recent study established that NtrX 
is phosphorylated in stationary phase in defined medium, as a result of acidification (48). In addition, NtrX appears to play a core role in regulating $C$. crescentus physiology, as $n t r X$ is essential for growth in complex medium and $\Delta n t r X$ cells grow more slowly than wild-type (WT) cells in defined medium (49).

In this study, we initially took a reverse genetic approach to characterize the role of ChvGI in regulating C. crescentus physiology. Deletion of $c h v G$ and $c h v I$ caused a distinctive growth defect in defined medium. By exploiting this defect, we identified striking genetic interactions between chvGI and $n t r Y, n t r X$, and $n t r Z$ (a previously uncharacterized gene). Epistasis analysis provided evidence that unphosphorylated $\mathrm{NtrX}$ is detrimental to cells lacking $c h v G$ or $c h v I$. We defined the ChvI transcriptional regulon and discovered that it overlaps significantly with genes regulated by

NtrX. In addition, we found that NtrZ promotes NtrX phosphorylation in vivo, likely by inhibiting NtrY phosphatase activity. We conclude that ChvGI and NtrYX interact at multiple transcriptional levels, working both in concert and in opposition to regulate $C$. crescentus growth in defined medium.

\section{Results}

\section{Loss of the ChvGI system limits growth in defined medium}

To investigate the physiological role of ChvGI in $C$. crescentus, we generated strains with inframe deletions of $c h v G$ or chvI and examined their growth. The deletion strains grew normally in complex medium (peptone-yeast extract, PYE), but they displayed a distinctive growth defect in defined medium (M2 minimal salts medium with xylose as carbon source, M2X) (Fig. 1A, $1 \mathrm{~B}$ and Fig. S1). Although overnight cultures of each strain inoculated from

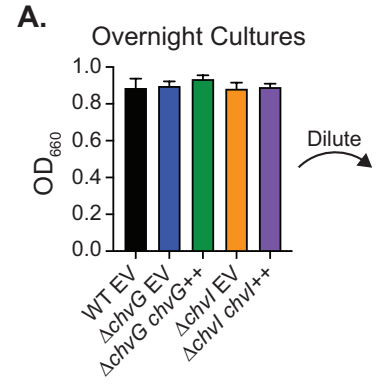

B.

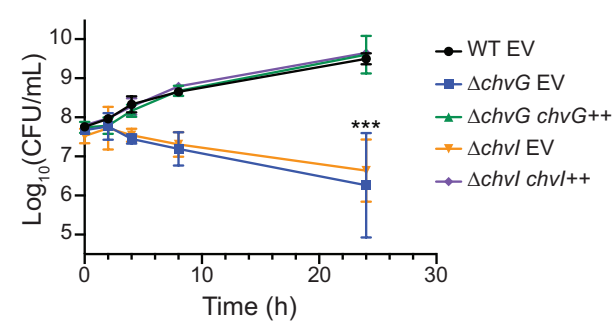

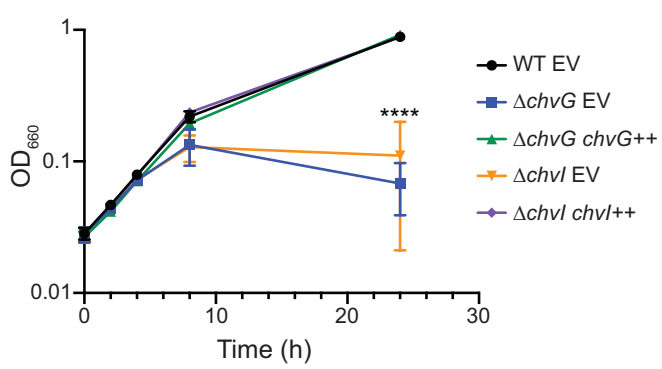

C.

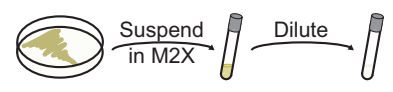

Figure 1: Loss of $c h v G$ or $c h v I$ limits culture density in defined M2X medium.

(A) Growth curves, measured by optical density $\left(\mathrm{OD}_{660}\right)$, of WT, $\Delta c h v G$, and $\Delta c h v I$ strains bearing empty vector (EV) or genetic rescue plasmid $(++)$ integrated at the xylose locus. Primary M2X cultures, inoculated from PYE plates, all grow to high density (left). However, upon back-dilution to $\mathrm{OD}_{660}=0.025, \Delta c h v G$ and $\Delta c h v I \mathrm{EV}$ strains saturate at a significantly lower OD (right). Points represent averages of three biological replicates $\pm \mathrm{SD} . * * * *=p<0.0001$, one-way ANOVA followed by Dunnett's post-test comparison to WT EV at $24 \mathrm{~h}$.

(B) Growth curves, measured by CFU, corresponding to the cultures in A. Points represent averages of three biological replicates \pm SD. $* * *=p<0.0005$, one-way ANOVA followed by Dunnett's post-test comparison to WT EV at $24 \mathrm{~h}$.

(C) Growth of cultures inoculated from PYE agar plates at different starting densities, measured by CFU. $\Delta c h v I$ cells carrying empty vector (EV) or genetic rescue plasmid (++) were suspended in M2X medium and immediately diluted to $\mathrm{OD}_{660}=0.001$ or 0.0001 . Points represent averages of three biological replicates $\pm \mathrm{SD} . *=p<0.05$, one-way ANOVA followed by Dunnett's post-test comparison to $\Delta c h v I \mathrm{EV}, 10^{-4}$ dilution at $30.5 \mathrm{~h}$. 
PYE agar plates grew to similar densities in M2X, strains lacking $c h v G$ or $c h v I$ exhibited reduced growth capacity after dilution, reaching a terminal density of only $\mathrm{OD}_{660} \sim 0.1$ (Fig. 1A). This lower cell density correlated with fewer colony-forming units (CFUs) (Fig. 1B). Ectopic overexpression of $\operatorname{ch} v G(\operatorname{chv} G++)$ or $\operatorname{ch} v I(\operatorname{ch} v I++)$ from a xylose-inducible promoter (xylose is the sole carbon source in M2X medium) fully rescued growth of $\Delta c h v G$ or $\Delta c h v I$ strains, respectively, under these conditions (Fig. 1A, 1B).

To evaluate if the growth defect was related to the number of cell divisions in defined medium, we resuspended cells in M2X from PYE agar plates and diluted cultures to several low starting densities. $\Delta c h v I \mathrm{EV}$ cultures started at $\mathrm{OD}_{660}=0.001$ and 0.0001 saturated at similar $\mathrm{CFU}$ values

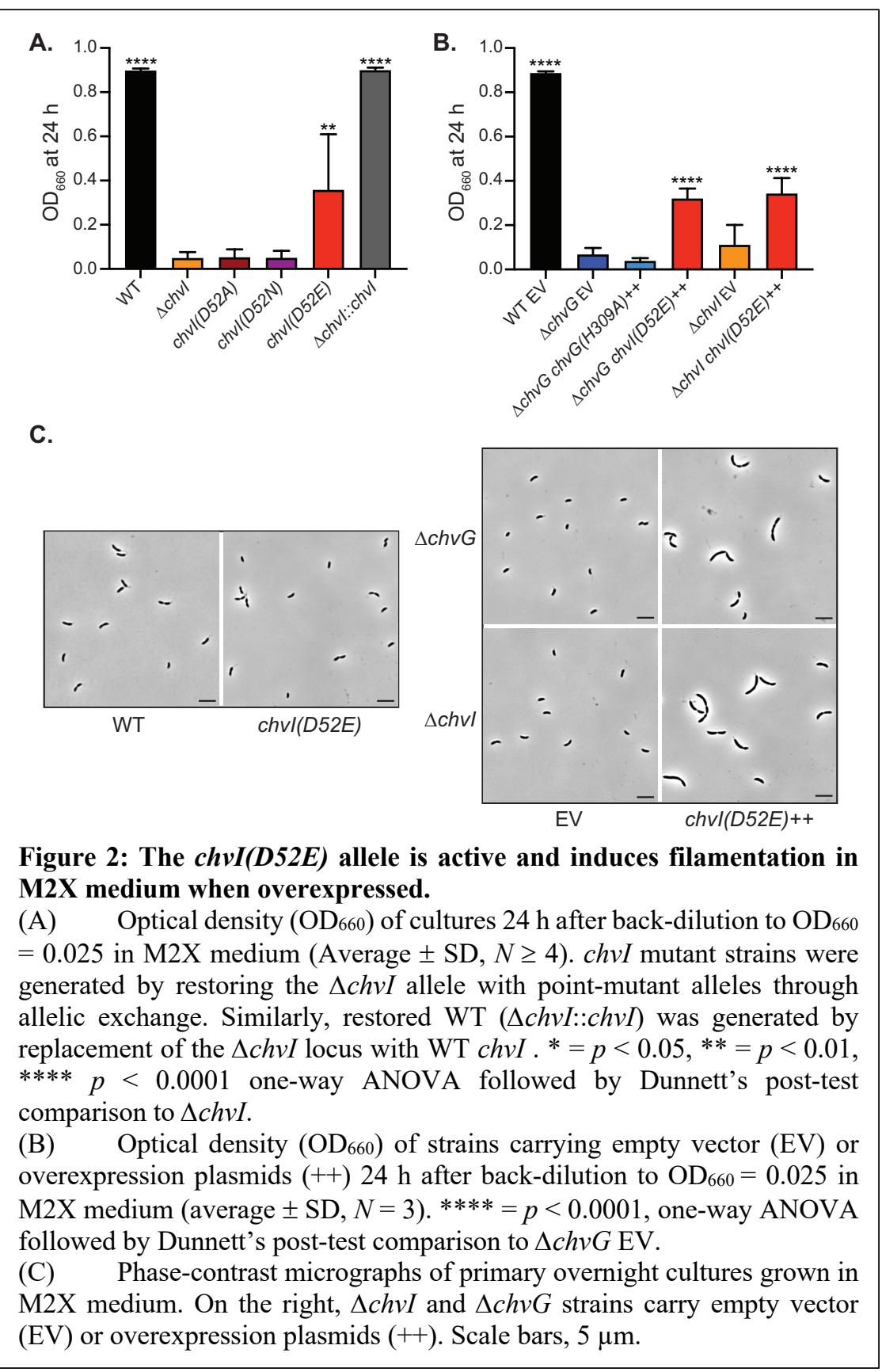

$\left(\sim 10^{7} \mathrm{CFU} / \mathrm{mL}\right)$ that were significantly lower than those of $\Delta c h v I \quad c h v I++$ cultures $\left(\sim 10^{9} \mathrm{CFU} / \mathrm{mL}\right)$ (Fig. 1C). Thus, $\Delta c h v I$ mutants that initiate from low density are not limited in the number of times they can divide in M2X medium, but rather reach a defined carrying capacity. $\Delta c h v I$ cultures started at higher densities $\left(\mathrm{OD}_{600}=0.05\right.$ and $0.1)$ grew to $\sim 10^{9} \mathrm{CFU} / \mathrm{mL}$, indicating that a high starting density enables primary overnight cultures to saturate at higher density (Fig. S2A).

Washing $\Delta c h v I$ cells once or twice with M2X before dilution had no effect on the number of CFUs at $30.5 \mathrm{~h}$, suggesting that trace contaminating PYE components do not contribute to growth in M2X primary cultures (Fig. S2B). Moreover, cell density alone did not determine the ability of $\Delta c h v I$ cultures to grow in $\mathrm{M} 2 \mathrm{X}$, as denser backdilutions from $\Delta c h v I$ overnight cultures did not reach higher viable cell 
counts (Fig. S2C). Together, our results suggest that the diminished growth capacity of $\Delta c h v I$ or $\Delta c h v G$ cells in M2X medium is a function of the time since resuspension from PYE plates and growth phase.

\section{ChvI phosphorylation is critical for growth in defined medium}

Given the similarity between the phenotypes displayed by $\Delta c h v I$ and $\Delta c h v G$ strains, we predicted that phosphorylation of ChvI might be important for growth in M2X medium. To test this hypothesis, we generated strains harboring $c h v I$ alleles encoding changes to the conserved sites of phosphorylation (D52A, D52N, and D52E). Growth of strains carrying the non-phosphorylatable alleles $\operatorname{ch} v I(D 52 A)$ and $\operatorname{ch} v I(D 52 N)$ was similar to $\Delta c h v I$ cells in M2X (Fig. 2A and Fig. S3A). By contrast, $\operatorname{ch} v I(D 52 E)$ cultures reached higher densities than $\Delta c h v I$ cultures, suggesting that ChvI(D52E) is active, albeit not to the same level as phosphorylated ChvI (Fig. 2A and Fig. S3A). Substitutions of the phosphorylatable aspartate with glutamate often act as phosphomimetic mutations and constitutively activate RRs (50-53). Thus, this result supports a critical role for ChvI phosphorylation during growth in M2X medium.

To further examine the importance of ChvI phosphorylation, we overexpressed $\operatorname{chvG}$ and $\operatorname{ch} v I$ alleles from a xylose-inducible promoter in knockout backgrounds. Although overexpression of $c h v G$ rescued growth of $\Delta c h v G$ cells (Fig 1B), overexpression of $c h v G(H 309 A)$, a catalytichistidine mutant, did not, indicating that phosphorylation of ChvG, and by extension ChvI, is important for growth in M2X medium (Fig. 2B). Overexpression of chvI(D52E) only partially rescued growth of $\Delta c h v I$ and $\Delta c h v G$ strains in $\mathrm{M} 2 \mathrm{X}$ and these strains grew similarly to the strain encoding $\operatorname{chvI}(D 52 E)$ at the native locus (Fig. 2A, 2B and Fig. S3B). However, in contrast to native expression of $\operatorname{chvI}(D 52 E)$, overexpression of this allele resulted in cell filamentation in $\mathrm{M} 2 \mathrm{X}$, but not PYE, consistent with a cell division defect (Fig. 2C and Fig. S3C). Thus, overexpression of phosphomimetic $\operatorname{ch} v($ D52E) appears to interfere with cell division and, perhaps as a result, only partially rescues growth in M2X medium.

\section{Mutations in $n t r Y, n t r X$, and a gene of unknown function, rescue the growth defect of $\Delta c h v I$ and $\Delta c h v G$ strains}

To better understand why ChvGI is critical for growth in defined medium, we employed a selection strategy to isolate second-site mutations that alleviate the growth defect of $\Delta c h v G$ and $\Delta c h v I$ cells. $\Delta c h v G$ and $\Delta c h v I \mathrm{M} 2 \mathrm{X}$-overnight cultures were back-diluted and grown until they reached high density, presumably due to proliferation of suppressor strains (Fig. 3A). We then isolated single colonies with WT-like growth in $\mathrm{M} 2 \mathrm{X}$ and used whole-genome sequencing to identify any acquired mutations (Fig. 3B).

In multiple independent strains, we identified nonsynonymous substitutions in the genes encoding the HK NtrY and its cognate RR NtrX (Fig. 3C). The ntrY mutations (L70H and A123V) are located in predicted transmembrane helices, which are involved in transmitting information from periplasmic sensing domains to the kinase domain (Fig. 3C) (54-56). We identified only one $n t r X$ allele, A19V, which lies in helix $1(\alpha 1)$ of the receiver domain (Fig. 3C). $\alpha 1$ is involved in the interaction interface between the HK dimerization and histidine phosphotransfer (DHp) domain and the RR REC domain $(57,58)$. We also note that the $n t r X(A 19 V)$ strain was isolated on PYE plates and appeared to grow normally in M2X, suggesting that this is not a complete loss-offunction allele (49). 
A.

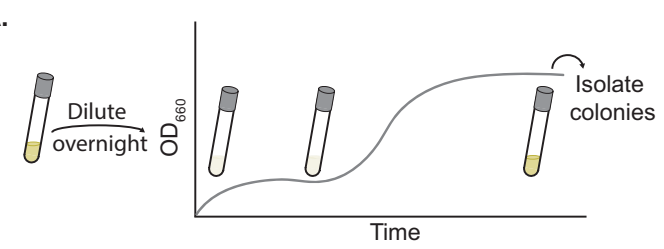

B.

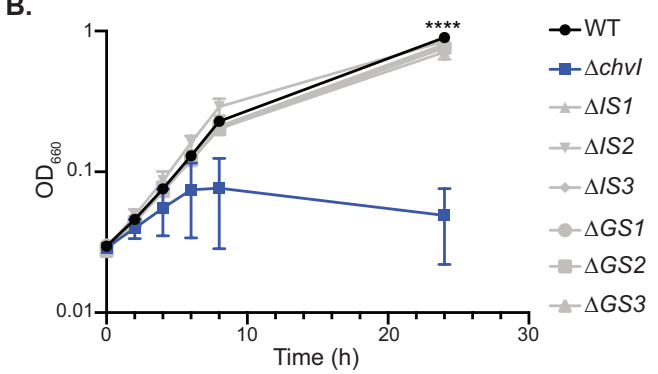

c.

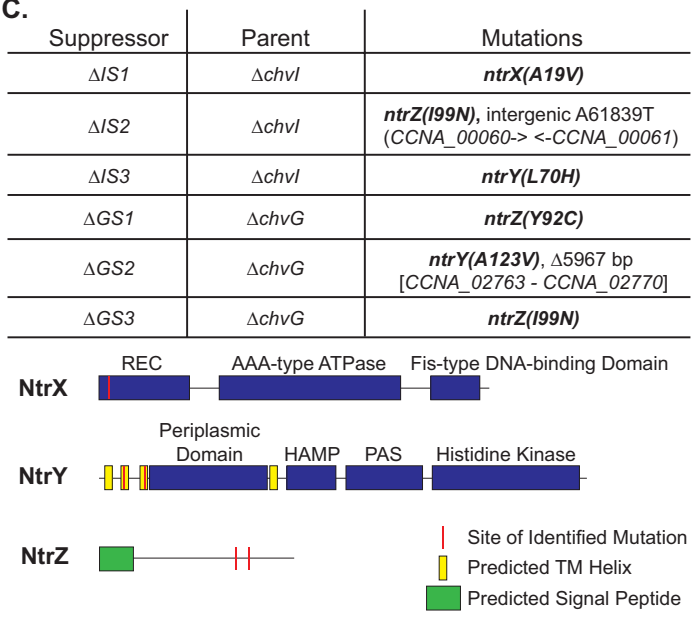

Figure 3: Mutations in the NtrYX TCS and a gene of unknown function suppress the growth defect of $\Delta c h v G$ and $\Delta c h v I$ strains in M2X medium.

(A) Schematic representation of the suppressor selection protocol. Primary overnight cultures in M2X medium were back-diluted to $\mathrm{OD}_{660}=0.025$ and grown until cultures grew to high turbidity. Single colonies were isolated for confirmation and sequencing.

(B) Growth curves, measured by optical density $\left(\mathrm{OD}_{660}\right)$, of WT, $\Delta c h v I$, and suppressor strains isolated from either the $\Delta c h v I$ strain $(\Delta I S \#)$ or the $\Delta c h v G$ strain $(\Delta G S \#)$ upon back-dilution in M2X medium. Points represent averages of three biological replicates \pm SD. $* * * *=p<0.0001$, one-way ANOVA followed by Dunnett's post-test comparison to $\Delta c h v I$ at $24 \mathrm{~h}$.

(C) Whole-genome sequencing results of each suppressor strain. The suppressor strain, parental strain, and identified polymorphism(s) are indicated. Mutations in $n t r Y$, ntrX, and ntrZ (CCNA_03863) are in bold. Domain structures of $\mathrm{NtrY}, \overline{\mathrm{N}} \operatorname{trX}$, and $\mathrm{NtrZ}$ are diagramed with domains in blue, signal peptides in green, transmembrane helices in yellow, and identified mutations in red.
In addition to mutations in $n \operatorname{tr} Y$ and $n t r X$, we identified three mutants with nonsynonymous substitutions in a gene encoding a putative periplasmic protein of unknown function (CCNA_03863, hereafter referred to as $n t r Z$ ) (Fig. 3C). The mutations identified (Y92C and $199 \mathrm{~N}$ ) are both located outside of the predicted signal sequence (Fig. 3C). Therefore, mutations in the NtrYX TCS and NtrZ appear to affect the growth of $\Delta c h v I$ and $\Delta c h v G$ cells in $\mathrm{M} 2 \mathrm{X}$ medium.

\section{Overexpression or deletion of $n t r X$} modulates the $\Delta c h v I$ growth phenotype

Several studies have noted similar phenotypes of chvGI and ntrYX mutants (25, 26, 41); however, to our knowledge none have established a genetic link between these TCSs. To better understand the connections between ChvGI and NtrYX in C. crescentus, we first characterized the genetic relationship between $c h v I$ and $n t r X$. Replacement of $n t r X$ with the $n \operatorname{tr} X(A 19 \mathrm{~V})$ allele restored growth of $\Delta c h v I$ cells in $\mathrm{M} 2 \mathrm{X}$ (Fig. 4A), ruling out other background mutations as causal for suppression of the $\Delta c h v I$ phenotype. However, overexpression of $n \operatorname{tr} X(A 19 \mathrm{~V})$ in the presence of the native $n t r X$ allele did not rescue growth of $\Delta c h v I$ cells (Fig. 4A). This result indicates that the $n \operatorname{tr} X(A 19 \mathrm{~V})$ allele is recessive, and suggests that the presence of WT NtrX contributes to the growth phenotype of $\Delta c h v I$ cells.

To examine this notion further, we tested the effect of deleting $n t r X$ in the $\Delta c h v I$ strain. $\Delta n t r X$ cells had a slight growth defect in M2X, but were clearly distinct from $\Delta c h v I$ cells (Fig. 4B). Deletion of $n t r X$ in a $\Delta c h v I$ background rescued growth, indicating that the presence of $n t r X$ is indeed detrimental to $\Delta c h v I$ cells (Fig. 4B). Suppression of the $\Delta c h v I$ growth phenotype was not due to a 
A.

B.
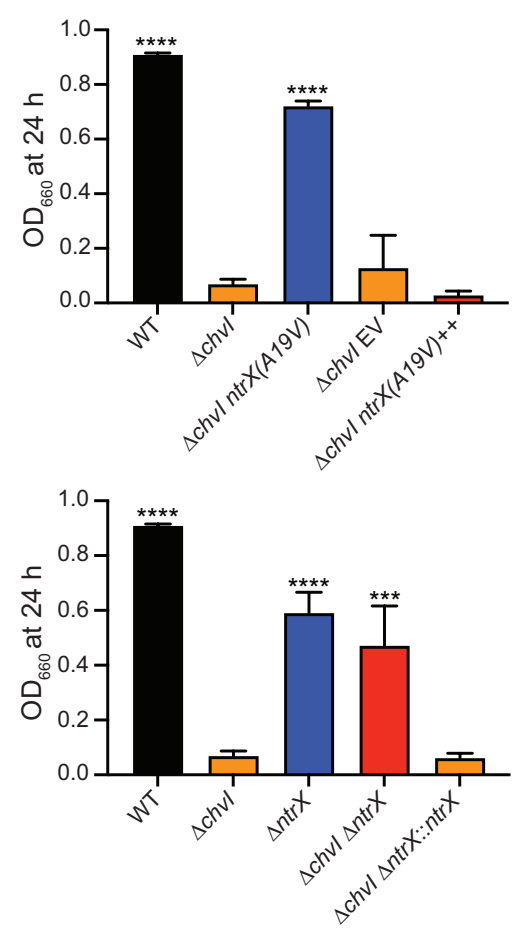

C.

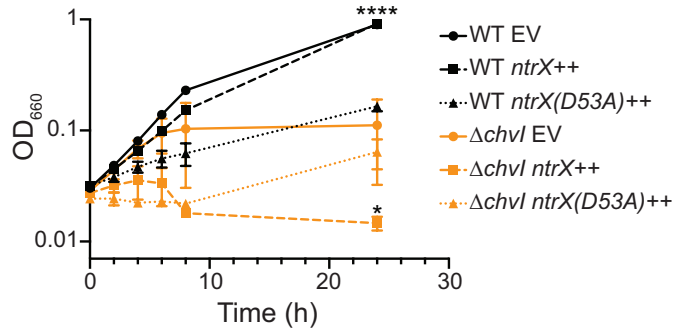

Figure 4: Non-phosphorylatable NtrX limits growth of cells in $\mathrm{M} 2 \mathrm{X}$ medium.

(A) Optical density $\left(\mathrm{OD}_{660}\right)$ of cultures $24 \mathrm{~h}$ after backdilution to $\mathrm{OD}_{660}=0.025$ in $\mathrm{M} 2 \mathrm{X}$ medium (average $\pm \mathrm{SD}, N$ $=3)$. The $n \operatorname{tr} X(A 19 V)$ allele was introduced by allele replacement for comparison with WT and $\Delta c h v I$ or by overexpression $(++)$ for comparison with an empty vector (EV) strain. $* * * *=p<0.0001$, one-way ANOVA followed by Dunnett's post-test comparison to $\Delta c h v I$.

(B) Optical density $\left(\mathrm{OD}_{660}\right)$ of cultures $24 \mathrm{~h}$ after backdilution to $\mathrm{OD}_{660}=0.025$ in $\mathrm{M} 2 \mathrm{X}$ medium (average $\pm \mathrm{SD}, N$ $=3)$. Restored $\Delta \operatorname{chvI}(\Delta \operatorname{chvI} \Delta n t r X:: n t r X)$ was generated by knocking $n t r X$ into the native $n t r X$ locus in $\Delta c h v I \Delta n t r X$ cells. $* * *=p<0.0005, * * * *=p<0.0001$, one-way ANOVA followed by Dunnett's post-test comparison to $\Delta c h v I$.

(C) Growth curves, measured by optical density $\left(\mathrm{OD}_{660}\right)$, of strains upon back-dilution in M2X medium. WT and $\Delta c h v I$ strains bear empty vector (EV) or $n t r X$ overexpression vectors $(++)$. Points represent averages of three biological replicates \pm SD. $*=p<0.05, * * * *=p<0.0001$, one-way ANOVA followed by Dunnett's post-test comparison to $\Delta c h v I \mathrm{EV}$ at $24 \mathrm{~h}$. second-s ite mutation, as restoration of the $n \operatorname{tr} X$ locus $(\Delta c h v I \quad \Delta n \operatorname{tr} X:: n \operatorname{tr} X)$ restored the growth defect in $\mathrm{M} 2 \mathrm{X}$ medium (Fig. 4B).

Given that the presence of $n t r X$ limits growth of $\Delta c h v I$ cells in M2X, we next tested whether overexpression of $n t r X$ affects growth of WT cells. Overexpression of $n t r X$ only moderately slowed growth of WT cells. However, overexpression of the nonphosphorylatable $n t r X(D 53 A)$ allele dramatically impaired the growth of WT cells in M2X, similar to the defect observed in $\Delta c h v I$ cells (Fig. 4C). Overexpression of either $n t r X$ or $n t r X(D 53 A)$ exacerbated the growth defect of $\Delta c h v I$ cells (Fig. $4 \mathrm{C}$ ). Together these results support a model in which unphosphorylated NtrX limits growth capacity in M2X medium, especially in $\Delta c h v I$ cells.

\section{NtrZ is a predicted periplasmic protein that functions upstream of NtrY to regulate levels of phosphorylated NtrX}

We next examined the nature of the $n t r Y$ and $n t r Z$ suppressor mutations. Overexpression of the $n t r Y$ and $n t r Z$ alleles identified in our suppressor strains restored growth of $\Delta c h v I$ cells in $\mathrm{M} 2 \mathrm{X}$ (Fig. 5A). In addition, overexpression of WT $n t r Y$, but not WT $n t r Z$, also significantly suppressed the $\Delta c h v I$ growth defect (Fig. 5A). We conclude that the $n t r Y$ and $n t r Z$ mutations are dominant, likely gain-offunction, alleles. As deletion of $n t r X$ rescued the growth of $\Delta c h v I$ cells, overexpression of $n t r Y$ or $n t r Z$ mutant alleles likely promotes phosphorylation and/or sequestration of NtrX. We attempted, but failed, to construct $\Delta c h v I$ $\Delta n t r Y$ and $\Delta c h v I \Delta n t r Z$ double-mutants 
trains, suggesting that deletion of either $n t r Y$ or $n t r Z$ is synthetically lethal with chvI deletion. This inability to isolate either double mutant also hinted that NtrY and NtrZ function in the same pathway. To test this possibility, we evaluated the phenotype of $n t r Y$ and $n t r Z$ deletions in M2X medium. However, the growth of both single deletions and the $\Delta n t r Y \Delta n t r Z$ double deletion was indistinguishable from WT, thus preventing epistasis analysis (Fig. 5B).

Phosphorylation of $\mathrm{NtrX}$ is likely important for stationary phase survival (48), and thus, we hypothesized that deletion of the $n t r Y$ $\mathrm{HK}$, and perhaps also $n t r Z$, might lead to a stationary-phase defect. Indeed, we observed significantly lower CFUs in both $\Delta n t r Y$ and $\Delta n t r Z$ cultures compared to WT cultures after $48 \mathrm{~h}$ of growth in M2X (Fig 5C). Notably, $\Delta n t r Y \Delta n t r Z$ cultures behaved similarly to the single mutants, suggesting that NtrY and $\mathrm{NtrZ}$ indeed function in the same pathway.

To place $n t r Y$ and $n t r Z$ relative to one another, we evaluated the ability of $n t r Y$ and $n t r Z$ alleles to rescue stationary-phase survival in the deletion strains. Both $\Delta n t r Y$ and $\Delta n t r Z$ cells were fully rescued by ectopic overexpression of each respective WT allele (Fig. 5D). Overexpression of $n \operatorname{tr} Y(L 70 H)$, but not ntrY, also fully rescued the phenotype of $\Delta n t r Z$ cells (Fig. 5D). By contrast, neither WT ntrZ nor $n t r Z(I 99 N)$ rescued $\Delta n t r Y$ cells (Fig.

5D). These data provide evidence that NtrZ functions upstream of NtrY, potentially as a kinase activator and/or phosphatase inhibitor.

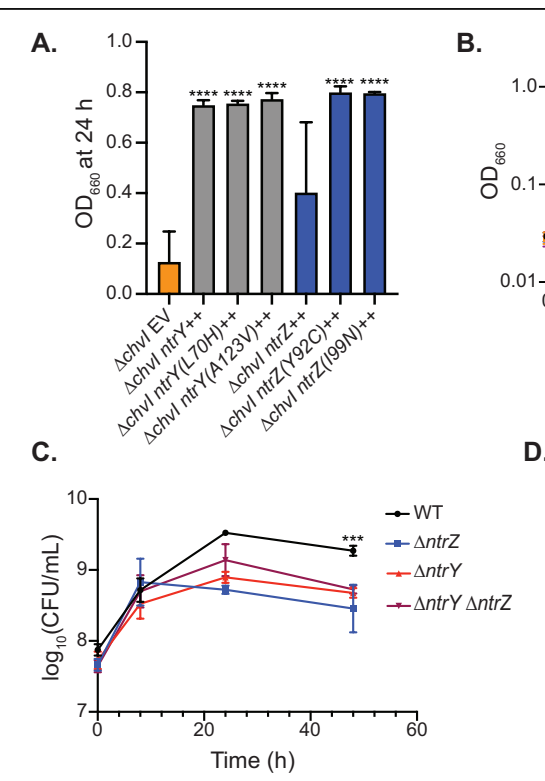

E.
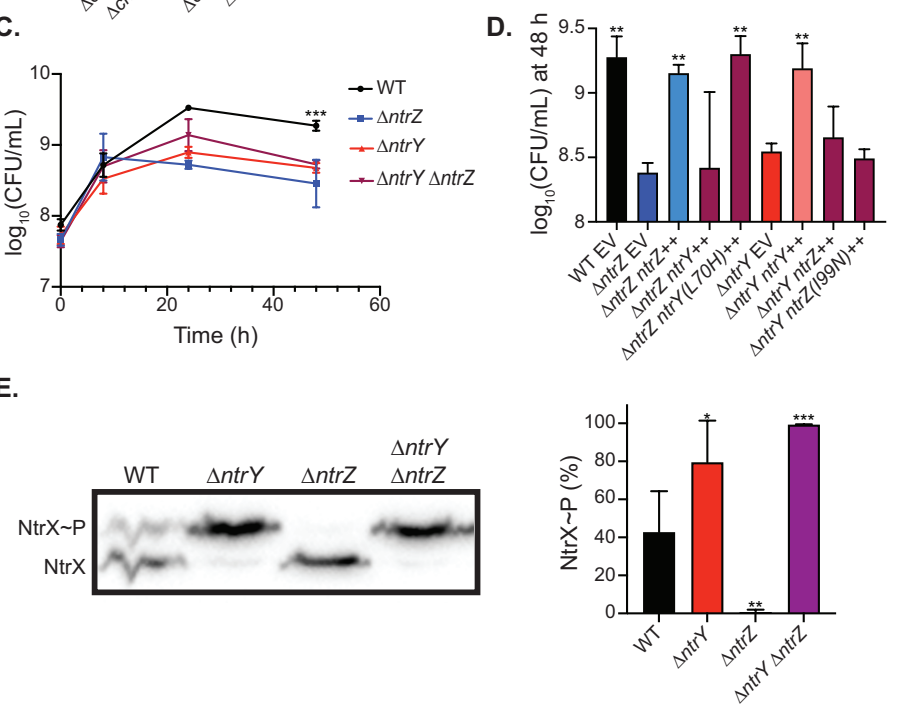

Figure 5: NtrZ functions upstream of NtrY as a phosphatase inhibitor. (A) Optical density $\left(\mathrm{OD}_{660}\right)$ of strains bearing empty vector $(\mathrm{EV})$ or $n t r Z$ and $n t r Y$ overexpression vectors ( ++$) 24 \mathrm{~h}$ after back-dilution to $\mathrm{OD}_{660}$ $=0.025$ in $\mathrm{M} 2 \mathrm{X}$ medium (average $\pm \mathrm{SD}, N=3$ ). $* * * *=p<0.0001$, one-way ANOVA followed by Dunnett's post-test comparison to $\Delta c h v I \mathrm{EV}$.

(B) Growth curves, measured by optical density $\left(\mathrm{OD}_{660}\right)$, of WT and knockout strains upon back-dilution in M2X medium. Points are averages of three biological replicates $\pm \mathrm{SD}$. $* * * *=p<0.0001$, one-way ANOVA followed by Dunnett's post-test comparison to WT at $24 \mathrm{~h}$.

(C) Growth curves, measured by CFU, for WT and mutant strains grown in M2X medium. Points are averages of three biological replicates \pm SD. ${ }^{* *}=p=0.0005$, one-way ANOVA followed by Dunnett's post-test comparison to $\Delta n t r Z$ at $48 \mathrm{~h}$.

(D) Cell density, measured by CFU, for $\Delta n t r Z$ and $\Delta n t r Y$ strains bearing empty vector (EV) or overexpression vectors $(++)$ at $48 \mathrm{~h}$ growth in $\mathrm{M} 2 \mathrm{X}$ medium. Points are averages of three biological replicates $\pm \mathrm{SD}$. $* *=$ $p<0.01$, one-way ANOVA followed by Dunnett's post-test comparison to $\Delta n t r Z \mathrm{EV}$ at $48 \mathrm{~h}$.

(E) Anti-HA western blot of lysates analyzed by Phos-tag gel electrophoresis (left). Each strain encodes $n t r X-H A$ at the native $n t r X$ locus. Phosphorylated (NtrX P) and unphosphorylated (NtrX) NtrX-HA are indicated. Quantification of the percent of NtrX that is phosphorylated (\% $\mathrm{NtrX} \sim \mathrm{P}$ ) (Right). Points are averages of four biological replicates $\pm \mathrm{SD}$. * = $p<0.05, * *=p<0.01, * * *=p<0.001$, one-way ANOVA followed by Dunnett's post-test comparison to WT.

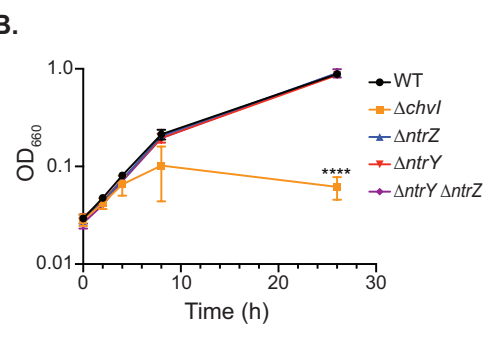


We next examined the phosphorylation state of NtrX directly using Phos-tag gel electrophoresis. To detect NtrX by western blotting, we constructed strains carrying C-terminally HA-tagged $n t r X$ ( $n \operatorname{tr} X-H A$ ) encoded at the native $n t r X$ locus. Phos-tag analysis of WT lysates at stationary phase revealed distinct bands for phosphorylated (NtrX P) and unphosphorylated (NtrX) NtrX-HA (Fig. $5 \mathrm{E}$ ). Deletion of $n t r Z$ ablated $\mathrm{NtrX} \sim \mathrm{P}$, consistent with a role for $\mathrm{NtrZ}$ in promoting $\mathrm{NtrX}$ phosphorylation (Fig. 5E). Surprisingly, $\Delta n t r Y$ cells displayed higher levels of NtrX phosphorylation than WT cells, suggesting that NtrY primarily acts as a phosphatase in vivo and is not required for NtrX phosphorylation. NtrZ indeed acts upstream of NtrY, as deletion of $n t r Z$ in the $\Delta n t r Y$ background did not affect NtrX phosphorylation (Fig. 5E). We conclude that NtrZ promotes NtrX phosphorylation by inhibiting NtrY phosphatase activity.

Our characterization of the $n t r Y, n t r X$, and $n t r Z$ mutants suggested that phosphorylation of NtrX may rescue the growth of $\Delta c h v I$ or $\Delta c h v G$ cells. NtrX is phosphorylated under acidic conditions, such as those encountered during stationary phase in M2G or M2X medium (48). Therefore, we tested whether the $\mathrm{pH}$ of $\mathrm{M} 2 \mathrm{X}$ affected the growth of $\Delta c h v I$ cultures. For both WT and $\Delta c h v I$ strains, primary overnight cultures diluted in $\mathrm{M} 2 \mathrm{X}$ at $\mathrm{pH} 7.0$ reached similar CFUs at $8 \mathrm{~h}$ as those diluted in standard $\mathrm{M} 2 \mathrm{X}$ ( $\mathrm{pH}$ 7.2) (Fig. 1B, S4). WT cultures were relatively unaffected by growth in M2X at $\mathrm{pH}$ 6.0, but had significantly fewer CFUs in M2X pH 5.5 vs. pH 7.0 (Fig. S4C). By contrast, $\Delta c h v I$ cells were markedly less fit in $\mathrm{M} 2 \mathrm{X} \mathrm{pH} 6.0$ than at $\mathrm{pH} 7.0$, and displayed an intermediate growth yield at $\mathrm{pH} 5.5$ relative to $\mathrm{M} 2 \mathrm{X}$ at either $\mathrm{pH} 6.0$ or $\mathrm{pH} 7.0$ (Fig. S4C). These results are consistent both with suppression of the $\Delta c h v I$ growth defect by NtrX phosphorylation and an important role for ChvGI in acid-stress responses (6).

\section{ChvI and NtrX regulate transcription of an overlapping set of genes}

Although ChvGI is known to regulate the expression of $c h v R$, the complete ChvI transcriptional regulon is not known. To examine the regulatory link between $n t r X$ and $c h v I$ in greater detail, we performed an RNA-seq experiment to comprehensively define ChvI-dependent gene regulation. As $\Delta c h v I$ cells grow poorly in M2X medium, we exploited overexpression of the phosphomimetic chvI(D52E) allele to assess ChvI-dependent transcription in PYE medium. Excluding the internal chvI control, we identified 162 genes with $>1.5$ fold-change in $\Delta \operatorname{chvI} \operatorname{ch} v I(D 52 E)++$ cells compared to $\Delta c h v I$ EV cells (Fig. 6A and Table S1). Of those, 140 were upregulated and 22 were downregulated, indicating that ChvI primarily serves as a transcriptional activator. Consistent with previous work, $c h v R$ was upregulated while $\operatorname{chvT}$ was downregulated by overexpression of $\operatorname{chvI}(D 52 E)(6,47)$. In addition, expression of both $\operatorname{chv} G$ and $h p r K$ was enhanced in cells expressing $\operatorname{ch} v I(D 52 E)$, pointing to positive autoregulation of the $c h v I G$ - $h p r K$ operon. We also observed regulation of multiple genes involved in envelope maintenance, metabolism, protein quality control, and transport (Fig. S5A). ChvI-dependent genes included multiple genes encoding proteases/peptidases (CCNA_01341, CCNA_02846, CCNA_01955, CCNA_02721, mmpA, CCNA_02594, CCNA_01121, CCNA_01202), peptidyl-prolyl and disulfide isomerases (CCNA_02889, CCNA_01654, CCNA_01759, CCNA_01653, CCNA_00378, CCNA_00379), members of the $\beta$-barrel assembly machine (BAM) complex (bamA, bamB, bamD, bamE, bamF), members of the Tol-Pal complex (tolB, $y b g F, t o l Q)$, and lipopolysaccharide biosynthesis genes (CCNA_01497, CCNA_03454,CCNA_01496,lpxC). Moreover, nearly 40\% of ChvI regulon genes encoded hypothetical proteins or proteins of unknown function (Fig. S5A). MEME analysis of the top 35 upregulated operons identified a putative ChvI binding motif, with GCC direct repeats 11 nucleotides apart, that closely resembles the recently characterized binding motif of Sinorhizobium meliloti ChvI (Fig. S5B) (5). 
A.

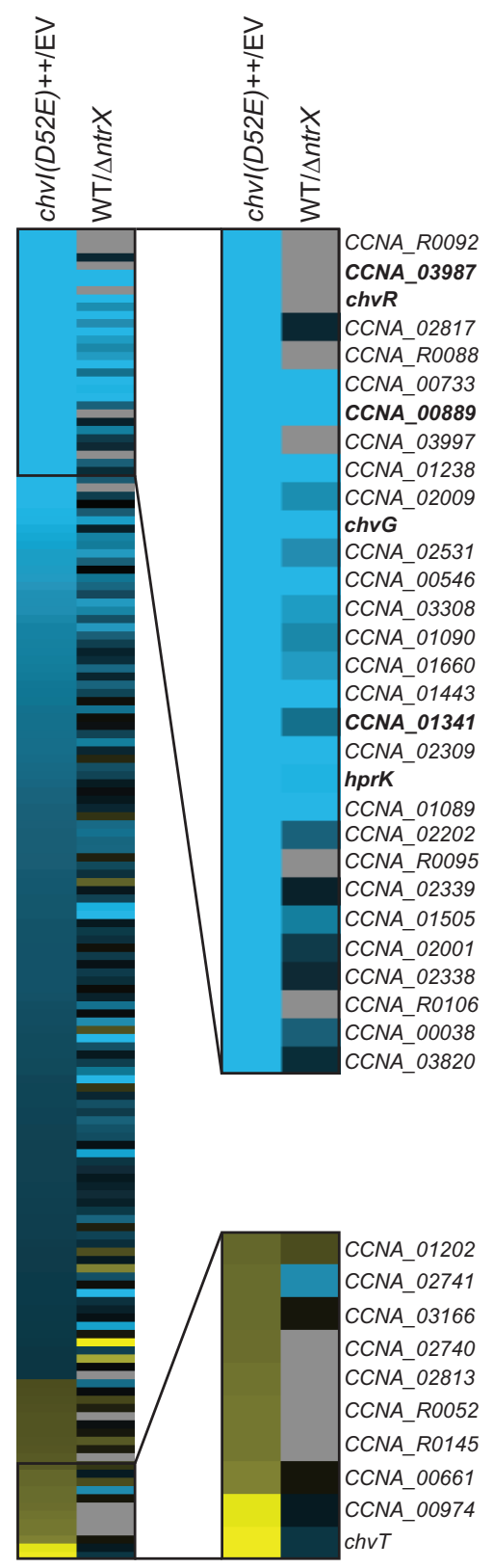

B.

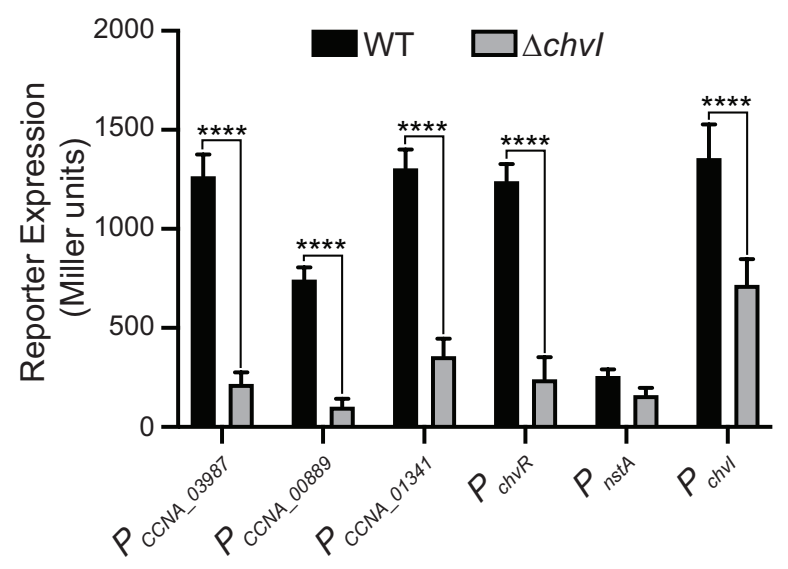

C.

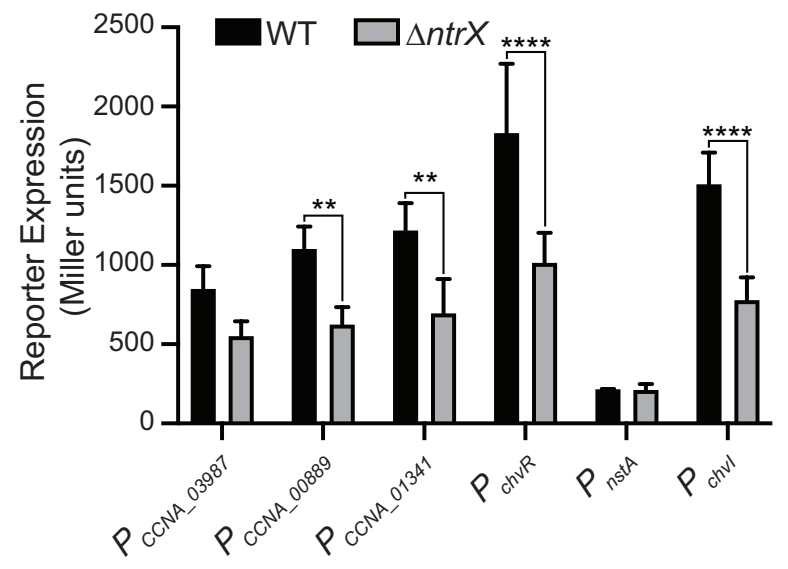

$\log _{2}$ (fold change)

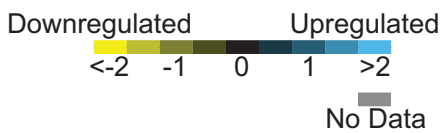

Figure 6: The ChvI and NtrX regulons overlap significantly.

(A) Heat map of $\log _{2}$ (fold change) for genes in the ChvI regulon (fold Change $>1.5$, FDR $p$-value $<0.05$ ) defined by RNA-seq $(\Delta c h v I \operatorname{ch} v I(D 52 E)++$ vs. $\Delta c h v I E V)$. $\log _{2}$ (fold change) is also shown for a microarray dataset comparing RNA levels in WT and $\Delta n t r X$ cells (49); grey cells indicate no data. The expression of upregulated genes (blue) is activated by ChvI or NtrX, whereas the expression of downregulated genes (yellow) is repressed by ChvI or NtrX. The genes most strongly regulated in the ChvI regulon are annotated (expression of those in bold is investigated further in $\mathrm{B}$ and $\mathrm{C}$ ).

(B) lacZ transcriptional reporter activity for a subset of genes in the ChvI regulon in WT and $\Delta c h v I$ strains in M2X medium (average $\pm \mathrm{SD}, N=3$ ). $* * * *=p<0.0001$, one-way ANOVA followed by Š́dák's post-test comparison for indicated pairs.

(C) lacZ transcriptional reporter activity for the same genes as in B in WT and $\Delta n t r X$ strains in M2X medium (average $\pm \mathrm{SD}, N=4)$. ${ }^{* *}=p<0.01, * * * *=p<0.0001$, one-way ANOVA followed by Š́dák’s post-test comparison for indicated pairs. 
As our regulon was defined by overexpression of a phosphomimetic ChvI mutant, we sought to validate our dataset under more physiological conditions. We constructed transcriptional $\beta$ galactosidase reporters for six selected regulon genes, from nstA (2-fold activation) to CCNA_03987 (128-fold activation). Strains carrying these transcriptional reporters were initially grown in PYE medium, followed by back-dilution and $4 \mathrm{~h}$ of growth in M2X medium. Consistent with previous reports, the reporter for the $c h v R$ promoter $\left(P_{c h v R}\right)$ was expressed in $\mathrm{M} 2 \mathrm{X}$ in a $c h v I-$ dependent manner (Fig. 6B) $(6,47)$. The remaining reporters, apart from $P_{\text {nsta, which was }}$ expressed at low levels, also exhibited clear $c h v I$ dependence, supporting our RNA-seq results (Fig. 6B). Unlike the other reporters, $P_{c h v I}$ was only 2-fold lower in $\Delta c h v I$ cells compared to WT cells, indicating that additional factors promote expression of the $c h v I G-h p r K$ operon.

We next compared our ChvI regulon with a previously published NtrX regulon that was determined using DNA microarrays (49). This experiment measured relative gene expression between $C$. crescentus WT (strain NA1000) and $\Delta n t r X$ cells during exponential growth in M2G medium, a condition where $\mathrm{NtrX}$ is expected to be largely unphosphorylated $(48,49)$. Surprisingly, a large fraction of the genes regulated by ChvI were also represented in the genes regulated by NtrX (Fig. 6A). That is, many of the genes upregulated by ChvI also appeared to be upregulated by NtrX (and likewise for downregulated genes). Using cutoffs of 1.5 fold-change for the ChvI RNA-seq data and 2.5 fold-change for the NtrX microarray data, we established that the ChvI regulon is significantly enriched for NtrX-dependent genes $(6.31$-fold enrichment, $p$-value $=8.99$ $\times 10^{-19}$, hypergeometric test). To confirm this overlap, we evaluated the effect of deleting $n t r X$ on our ChvI regulon reporters. Strains carrying the transcriptional reporters were grown to log phase $\left(\mathrm{OD}_{660} \sim 0.1-0.2\right)$ in $\mathrm{M} 2 \mathrm{X}$ medium before assaying $\beta$-galactosidase activity. Four of the six reporters exhibited $n t r X$ dependence, including two genes (CCNA_00889 and $c h v R)$ not evaluated in the NtrX microarray experiment (Fig. 6C). We note that transcription from the chvI promoter $\left(P_{c h v I}\right)$ was activated by both $n t r X$ and $c h v I$, raising the possibility that $\mathrm{NtrX}$ may indirectly affect ChvI-dependent genes via upregulation of $c h v I G-h p r K$.

Given the oppositional nature of ChvI and NtrX during growth in M2X medium, we were surprised to see such a high degree of similarity in the genes they regulate. However, a small subset of genes exhibited opposite regulation by ChvI and NtrX, which might therefore account for suppression of the $\Delta c h v I$ growth defect by deletion of $n t r X$ (Fig. S6A). We overexpressed (for those genes upregulated by ChvI) or knocked out (for those genes downregulated by ChvI) each of these genes in $\Delta c h v I$ cells and tested their growth capacity in M2X medium. Only one candidate, $c h v T$, had any effect on $\Delta c h v I$ cells. Specifically, deletion of $c h v T$ partially rescued growth of $\Delta c h v I$ cells in M2X (Fig. 7A and Fig. S6B). Differential regulation of $c h v T$ expression by ChvI and NtrX may therefore contribute to the growth defect observed in M2X medium.

\section{Discussion}

\section{The importance of the $C$. crescentus ChvGI system for growth in defined medium}

To our knowledge, the only reported physiological phenotype for chvGI mutants in C. crescentus is sensitivity to the antibiotic vancomycin (47). However, previous work indicated that ChvGI might be important for growth in defined medium (6). In fact, chvGI mutants in other $\alpha$ - 


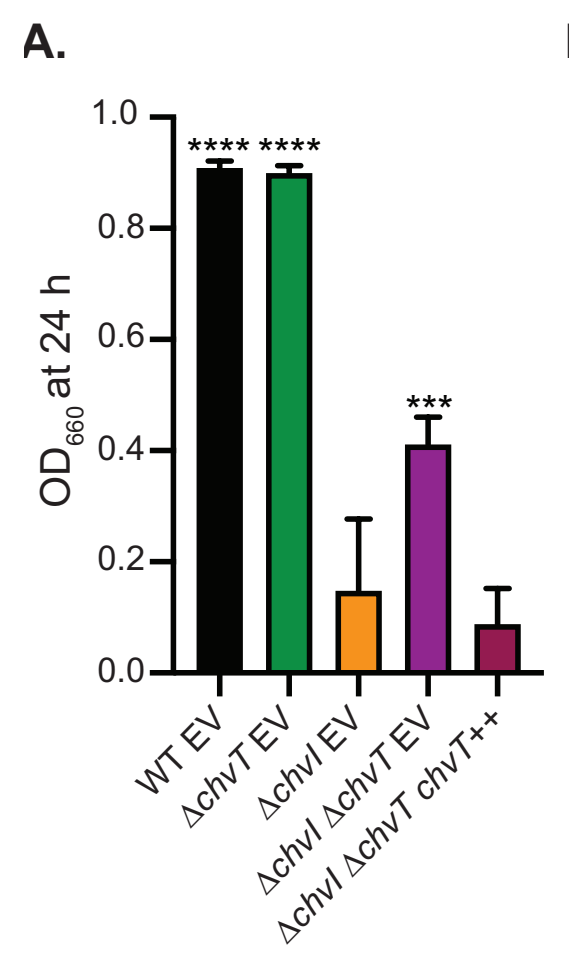

B.

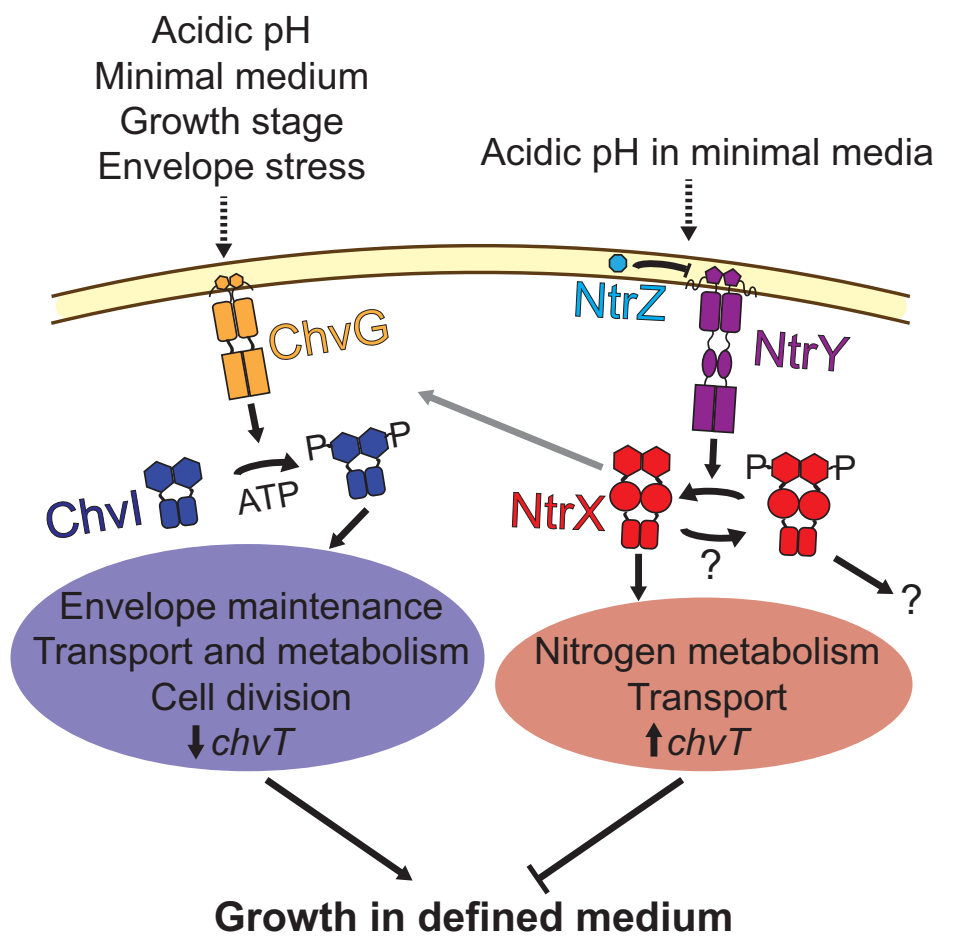

Figure 7: Deletion of $c h v T$ partially restores growth of $\Delta c h v I$ cells in $\mathrm{M} 2 \mathrm{X}$ medium.

(A) Optical density $\left(\mathrm{OD}_{660}\right)$ of WT, $\Delta$ chvT, and $\Delta c h v I \Delta c h v T$ strains, with empty vector (EV) or $c h v T$ overexpression vector $(++), 24 \mathrm{~h}$ after back-dilution to $\mathrm{OD}_{660}=0.025$ in $\mathrm{M} 2 \mathrm{X}$ medium (average $\pm \mathrm{SD}, N=4$ ). $* * *$ $=p<0.0005, * * * *=p<0.0001$, one-way ANOVA followed by Dunnett's post-test comparison to $\Delta c h v I \mathrm{EV}$.

(B) Proposed model for the regulatory interactions between ChvGI and NtrYXZ. ChvG (orange) is activated by a variety of cellular conditions (dashed arrow) and phosphorylates ChvI (blue). Phosphorylated ChvI regulates genes involved in envelope maintenance, transport, metabolism, and cell division (blue oval). In addition, ChvI represses expression of $c h v T$. NtrY (purple) is repressed by NtrZ under acidic conditions in defined medium (dashed arrow), reducing dephosphorylation of NtrX (red). Unphosphorylated NtrX regulates much of the ChvI regulon, likely via upregulation of the $c h v I G$ - $h p r K$ operon (grey arrow). In addition, unphosphorylated NtrX upregulates $c h v T$ and regulates expression of genes involved in nitrogen metabolism and transport (red oval). Phosphorylated ChvI and unphosphorylated NtrX oppose each other in regulating growth in defined medium, partially via differential regulation of $c h v T$. The transcriptional role of phosphorylated NtrX and source of phosphorylation (question marks) are not yet known.

proteobacteria are sensitive to nutritional conditions, although most, with the exception of Brucella abortus, exhibit particularly poor growth in complex media $(4,8,27,59)$. Deletion of $c h v G$ or $c h v I$ in $C$. crescentus caused a distinctive growth defect in $\mathrm{M} 2 \mathrm{X}$ medium. $\Delta c h v I$ cultures do grow in M2X upon inoculation from PYE plates, suggesting that the physiological state of the cell in PYE agar is initially amenable to growth in defined medium (Fig. 1). However, this tolerance is limited by time, with only higher inocula able to reach high cell density (Fig. 1 and Fig. S2). Why then, can primary overnight cultures persist at high density until back-dilution in fresh M2X? One possibility is that the low $\mathrm{pH}$ of $\mathrm{M} 2 \mathrm{X}$ at stationary phase preserves $\Delta c h v I$ and $\Delta c h v G$ cells, potentially by triggering phosphorylation of NtrX (Fig. 7B) (48). This model is supported by the observation that $\Delta c h v I$ cells reach higher CFUs when back-diluted in $\mathrm{M} 2 \mathrm{X}$ at $\mathrm{pH} 5.5 \mathrm{vs}$. $\mathrm{pH} 6.0$ (Fig. S4C). As $\sim 20 \%$ of the NtrX pool is phosphorylated in $\mathrm{M} 2 \mathrm{G}$ medium at $\mathrm{pH} 6.0 \mathrm{vs}$. $\sim 40 \%$ at pH 5.5 (by Phos-tag analysis), the $\mathrm{pH}$ 6-5.5 transition would significantly change the level of 
unphosphorylated $\operatorname{NtrX}(48)$. However, as $\Delta c h v I$ and $\Delta c h v G$ cells also appear to be sensitive to acidic $\mathrm{pH}$, additional factors are likely at play (Fig. S4C). For example, several extracytoplasmic function (ECF) sigma factors are involved in resistance to stationary-phase stress and might play a protective role in $\Delta c h v I$ and $\Delta c h v G$ cells (60-62).

A recent study failed to identify chvG or $c h v I$ as being important for fitness in M2X medium (63). However, in this work M2X cultures were inoculated from PYE starter cultures at high enough density to reach saturation in 5 doublings. Thus, these experiments likely mimicked our primary overnight cultures, obscuring detection of fitness defects for chvGI mutants.

\section{The ChvI transcriptional regulon in $C$. crescentus}

Although ExoR or ChvI transcriptional regulons have been defined in several $\alpha$-proteobacteria (4, $5,26,64)$, the $C$. crescentus ChvI regulon was unknown prior to this work. We employed RNAseq to detect direct and indirect transcriptional targets of ChvI in C. crescentus (Fig. 6, Table S1). Our ChvI regulon contained several classes of genes noted in other $\alpha$-proteobacteria, including those encoding outer membrane proteins and transporters, metabolic enzymes, lipopolysaccharide biosynthesis enzymes, and stress response proteins $(4,5,26,64)$. We note, in particular, that the C. crescentus ChvI regulon contained a large number of genes encoding proteins involved in envelope maintenance, including nearly the entire BAM complex, the envelope integrity protein EipA, members of the Tol-Pal complex, and a variety of chaperones and proteases (Fig. 6 and Table S1) (65-68). The idea that ChvGI is involved in envelope integrity is consistent with previous observations that ChvGI is activated by envelope stress and confers resistance to antibiotics targeting the cell wall (47). However, $\sim 40 \%$ of genes regulated by ChvI are annotated generically or as hypotheticals, and thus, more work will be required to characterize the pathways downstream of the ChvGI system. In $A$. tumefaciens, $S$. meliloti, and C. crescentus, ChvGI appears to suppress cell motility and/or chemotaxis $(5,15,20,26,69,70)$. However, unlike in A. tumefaciens and $S$. meliloti, $C$. crescentus ChvI does not regulate any obvious flagellar or chemotaxis genes, suggesting that effects on motility may be due to post-transcriptional regulation and/or alterations in cell-cycle progression $(5,26,71-73)$.

Overexpression of the phosphomimetic $\operatorname{chvI}(D 52 E)$ allele induced cell filamentation in $\mathrm{M} 2 \mathrm{X}$, implicating ChvI in regulating cell division, and cytokinesis in particular. ChvI upregulates several genes related to cell division, including zauP, members of the Tol-Pal complex, smc, and nstA (68, 74-77). We note that overexpression of a proteolytically stable mutant form of nstA induces cell filamentation and both zauP and the Tol-Pal complex are involved in regulating cytokinesis (74, 77). Perhaps over-induction of these regulon genes, in combination with the cellular state in M2X medium, interferes with proper cell division.

\section{Genetic interactions between ChvGI and NtrYX}

The severe growth defect of $\Delta c h v G$ and $\Delta c h v I$ cells in M2X medium allowed us to uncover the first known genetic interaction between $c h v G I$ and $n t r Y X$ (Fig. 3). Importantly, deletion of $n t r X$ suppressed the growth defect of $\Delta c h v I$ cells, whereas overexpression of non-phosphorylatable $n t r X(D 53 A)$ was deleterious for growth in both $\Delta c h v I$ and WT cells (Fig. 4). These results suggest that the activity of unphosphorylated $\mathrm{NtrX}$ is particularly detrimental in cells lacking chvI. Although unphosphorylated response regulators are often assumed to be inactive, multiple RRs are known to affect transcription in their unphosphorylated states (78-82). Thus, we propose that 
phosphorylated ChvI and unphosphorylated NtrX oppose each other to regulate growth in defined medium (Fig. 7B). We note that a past study examining connections between ChvI and NtrX in $S$. meliloti did not test whether perturbations in NtrYX signaling might affect $\Delta c h v I$ phenotypes (25). We predict that a similar ChvI-NtrX relationship may be conserved in other $\alpha$-proteobacteria.

As gain-of-function mutations in $n t r Y$ and $n t r Z$ suppress the growth defect of $\Delta c h v I$ cells, we also propose that phosphorylation of NtrX relieves its detrimental activity, possibly by changing its transcriptional regulon. Although the global transcriptional effects of NtrX phosphorylation have yet to be characterized, in vitro phosphorylation of $B$. abortus $\mathrm{NtrX}$ does induce conformational changes and alters, but does not weaken, binding to the $n \operatorname{tr} Y X$ promoter (83). Interestingly, we were unable to construct $\Delta c h v I \Delta n t r Y$ and $\Delta c h v I \Delta n t r Z$ strains, suggesting that these gene deletion combinations are synthetically lethal in PYE. As $n t r X$ is also essential in PYE (20), the balance between unphosphorylated and phosphorylated NtrX may be important for growth in both complex and defined media.

To identify downstream genes that mediate the oppositional relationship between ChvI and NtrX, we compared their transcriptional regulons (Fig. 6 and Table S1). The ChvI regulon strongly overlapped with that of NtrX, as $80 \%$ of the top $30 \mathrm{ChvI}$-activated genes are also activated by $\mathrm{NtrX}$ (per our $>1.5$-fold cutoff). By contrast, only $20 \%$ of the top $30 \mathrm{NtrX}$-activated genes exhibit $>1.5$-fold ChvI-dependence (49). Given that $c h v I, c h v G$, and $h p r K$ are upregulated by NtrX, it may be the case that NtrX simply activates expression of the $c h v I G-h p r K$ operon, thereby altering transcription of genes in the ChvI regulon (Fig. 7B, grey arrow). However, further work is required to define the mechanism by which NtrX affects the ChvI regulon, as it may also indirectly affect ChvGI signaling or directly regulate expression of regulon genes. The overlap we observed between the ChvI and NtrX regulons may be restricted to C. crescentus and close relatives, as chvG and chvI are not transcriptionally regulated by NtrYX in Rhodobacter sphaeroides (40). However, ChvGI and NtrYX may still be transcriptionally linked in more distantly-related $\alpha$ proteobacterial species, as $n t r X$ is part of the A. tumefaciens ExoR regulon (26).

The majority of overlapping genes in the ChvI and NtrX regulons cannot account for the detrimental effect of unphosphorylated NtrX on $\Delta c h v I$ cells. Therefore, we focused on eight genes that exhibited opposing transcriptional regulation by ChvI and NtrX (Fig. S6). Given that NtrX largely reinforces the ChvGI TCS, these oppositional effects may reflect direct transcriptional regulation by NtrX or the effects of unique NtrX regulon genes. Only deletion of $\operatorname{ch} v T$ improved the growth of $\Delta c h v I$ cells, indicating that suppression of $c h v T$ RNA levels by ChvI may be important for growth in M2X medium (Fig. 7). chvT is linked to diverse phenotypes in $C$. crescentus, including survival in stationary phase, sensitivity to cell wall-targeting antibiotics, and sensitivity to bacteriocins $(47,84,85)$. However, the molecule(s) transported by ChvT remain undefined. High ChvT levels may contribute to defects in transport in defined medium and/or alter membrane integrity, impacting the viability of cells lacking ChvGI (47). It is clear, though, that altered $c h v T$ expression cannot fully explain the growth deficiency of $\Delta c h v I$ cells in M2X medium. This growth defect may result from the collective action of several genes, and therefore, manipulation of individual candidates may not rescue growth in M2X. Moreover, 15 genes in the ChvI regulon are absent from the NtrX microarray dataset, raising the possibility that one or more may be differentially regulated by ChvI and NtrX (Fig. 6, Table S1). ChvI and NtrX might also 
interact more indirectly, as each regulates unique subsets of genes that may affect growth in M2X medium.

\section{On the role of NtrZ}

NtrYX is associated with a wide range of physiological responses, from nitrogen metabolism to redox sensing and cell envelope maintenance $(25,35,39,40)$. Despite these phenotypic observations, little is known about NtrY activity in vivo and the regulation of NtrY via its periplasmic domain. Our work reveals a surprising phosphatase pathway involving the previously uncharacterized protein NtrZ. Phos-tag analysis of NtrX phosphorylation in vivo clearly demonstrates that NtrY is dispensable for NtrX phosphorylation, and suggests that NtrY primarily acts as an NtrX phosphatase (Fig. 5). In addition, NtrZ appears to inhibit NtrY phosphatase activity, as deletion of $n t r Z$ abolishes $N \operatorname{tr} X$ phosphorylation only when $n t r Y$ is present (Fig. 5). Thus, we propose that NtrZ inhibits NtrY phosphatase activity, stabilizing the pool of phosphorylated $\mathrm{NtrX}$ (Fig. 7). In the future, we are interested in determining whether NtrZ physically interacts with the NtrY periplasmic domain or affects NtrY activity indirectly. Several known periplasmic or membrane-bound TCS regulators directly interact with HK periplasmic domains (33, 86-88). Although our model does not exclude the possibility that NtrY phosphorylates NtrX under certain conditions, there is clearly another source of NtrX phosphorylation in $C$. crescentus. An additional HK may phosphorylate NtrX, although the most likely candidate, NtrB, does not phosphorylate NtrX in vitro (49). Alternatively, some metabolic intermediates, such as aspartyl-phosphate or carbamoyl-phosphate, can serve as phosphodonors for RRs in vivo and in vitro $(1,89-92)$.

Interestingly, we initially placed $n t r Z$ upstream of $n t r Y$ by examining the similar stationary-phase survival phenotypes of $\Delta n t r Y$ and $\Delta n t r Z$ cells (Fig. 5). Given our Phos-tag results, these phenotypes suggest that both phosphorylated and unphosphorylated NtrX play important roles in stationaryphase survival. This idea is consistent with the fact that NtrX phosphorylation increases upon entry into stationary-phase, but eventually decreases (48).

Fernández et al. reported that NtrX phosphorylation is triggered by acidic $\mathrm{pH}$ in defined medium (48). Therefore, we hypothesize that inhibition of NtrY by NtrZ may be enhanced by acidic $\mathrm{pH}$. Thus, NtrZ and ExoR potentially share a regulatory theme, in which their activities towards their respective HKs are affected by low $\mathrm{pH}(16,33,34)$. However, it remains to be seen if, as with ExoR, proteolysis plays a role in controlling NtrZ-dependent regulation of NtrY (16). Given the results of our suppressor selection, it would appear that mutations in NtrZ or in the transmembrane helices of NtrY may bypass regulation by acidic $\mathrm{pH}$. In an alignment of $100 \mathrm{NtrZ}$ homologs, ranging from $41 \%$ to $100 \%$ sequence identity, the $\mathrm{Y} 92$ position is conserved as an aromatic residue $(95 \% \mathrm{Y})$ while $\mathrm{I} 99$ is conserved as an aliphatic hydrophobic residue $(51 \% \mathrm{I}$, common $\mathrm{L}$ and $\mathrm{V}$ substitutions), suggesting that these residues are important for NtrZ function. Further work is required to determine how mutation of Y92 or 199 may alter interaction with the NtrY periplasmic domain or affect a different aspect of NtrZ function. An alignment of the non-cytoplasmic portion of $250 \mathrm{NtrY}$ sequences, ranging from $37 \%$ to $100 \%$ identity, reveals that the L70 position is largely conserved as an aliphatic hydrophobic residue (94\% L) whereas A123 is conserved as a hydrophobic residue $(93 \% \mathrm{~A})$, albeit with occasional bulky-aromatic substitutions. It is unsurprising, then, that the $\mathrm{L} 70 \mathrm{H}$ and $\mathrm{A} 123 \mathrm{~V}$ substitutions appear to affect NtrY function. In fact, 
several studies have identified transmembrane mutations that alter HK kinase and phosphatase activities (93-95).

Unlike the periplasmic kinase regulator ExoR, which contains Sel1-like repeats typically involved in protein-protein interactions, NtrZ does not contain any conserved domains or motifs (95). Moreover, NtrZ appears restricted to the order Caulobacterales, albeit with a few distant homologs in other $\alpha$-proteobacteria. Notably, neither B. abortus, S. meliloti, nor A. tumefaciens contain NtrZ homologs, and conservation of the non-cytoplasmic region of NtrY is quite low between $C$. crescentus and these organisms (24-27\% identity). However, L70 is largely conserved (L or V) and A123 is entirely conserved between these NtrY homologs. Thus, the L70H and A123V substitutions may have similar effects on NtrY activity in these organisms, and further analyses of these mutants may shed light on a conserved NtrY activity switch. Moreover, it will be interesting to see if NtrY also primarily acts as a phosphatase and/or is regulated by periplasmic effectors in S. meliloti, A. tumefaciens, and B. abortus.

\section{Materials and Methods Strains and Plasmids}

All plasmids were constructed using standard molecular biology techniques. See Table S2 for strain, plasmid, and primer information. Plasmids for generating in-frame deletions and allelereplacements were generated by cloning homologous upstream and downstream regions into pNPTS138. Transcriptional reporter plasmids were generated by cloning 400-500 bp upstream of the open reading frame into pRKlac290. For overexpression strains, ORFs were inserted into pMT585, a plasmid for xylose-inducible expression that integrates at the $x y l X$ locus. Plasmids were transformed into C. crescentus CB15 strain by electroporation or tri-parental mating. In-frame deletion and allele-replacement strains were generated by a double recombination strategy involving $s a c B$ counterselection on PYE plates supplemented with $3 \%$ sucrose (97). For $\Delta n t r X$ knockout strains, counterselection was carried out on $\mathrm{M} 2 \mathrm{X}+0.5 \%$ sucrose plates due to their growth defect on PYE. $\Delta n t r X$ and $\Delta c h v I \Delta n t r X$ strains were grown only on M2 medium. Construction of $\Delta c h v I \Delta n t r Y$ and $\Delta c h v I \Delta n t r Z$ mutants was attempted using both PYE and M2X counterselection methods. All $C$. crescentus strains were grown at $30^{\circ} \mathrm{C}$. For strains carrying pRKlac290 plasmids, oxytetracycline was added to $1 \mu \mathrm{g} / \mathrm{mL}$ in liquid and $2 \mu \mathrm{g} / \mathrm{mL}$ in PYE agar or $1 \mu \mathrm{g} / \mathrm{mL}$ in $\mathrm{M} 2 \mathrm{X}$ agar.

M2X medium contained $6.1 \mathrm{mM} \mathrm{Na} 2 \mathrm{HPO}_{4}, 3.9 \mathrm{mM} \mathrm{KH}_{2} \mathrm{PO}_{4}, 9.3 \mathrm{mM} \mathrm{NH} 4 \mathrm{Cl}, 0.25 \mathrm{mM} \mathrm{CaCl}_{2}$, $0.5 \mathrm{mM} \mathrm{MgSO}_{4}, 1: 1000$ 100X Ferrous Sulphate/Chelate Solution (Sigma), and 0.15\% xylose. PYE medium contained $0.2 \%$ peptone, $0.1 \%$ yeast extract, $0.5 \mathrm{mM} \mathrm{CaCl}_{2}$, and $1 \mathrm{mM} \mathrm{MgSO}_{4}$.

\section{Measurement of Growth in M2X Medium}

Primary M2X cultures were inoculated from plates to an approximate density of $\mathrm{OD}_{660} 0.02-0.10$ and grown overnight. Overnight cultures were back-diluted to $\mathrm{OD}_{660}=0.025$ and $\mathrm{OD}_{660}$ was recorded at the indicated times. To enumerate colony forming units, samples were taken at the indicated time points and 10-fold serial dilutions were plated on PYE agar. For pH experiments, $\mathrm{M} 2 \mathrm{X}$ medium was adjusted to the indicated $\mathrm{pH}$ using $\mathrm{HCl}$.

For experiments with controlled starting densities, cells were resuspended in M2X medium directly from PYE plates. These resuspensions were then diluted to the indicated $\mathrm{OD}_{660}$ and the 
cultures were titered for CFUs. In washing experiments, $1 \mathrm{~mL}$ resuspended cells were spun down at $8 \mathrm{k} \times g$ for $3 \mathrm{~min}$ and resuspended in $1 \mathrm{~mL}$ fresh M2X medium once (1X) or twice (2X) before dilution. Plotting and statistical analyses were carried out using Prism (Graphpad).

\section{Microscopy}

Samples of $\Delta c h v G$ and $\Delta c h v I$ cells were taken from overnight M2X or PYE $+0.15 \%$ xylose cultures and imaged with a DMI6000B (Leica) microscope in phase contrast using a HC PL APO $63 \mathrm{x} / 1.4$ na oil $\mathrm{Ph} 3 \mathrm{CS} 2$ objective. Images were captured using an Orca- $\mathrm{R}^{2} \mathrm{C} 10600$ digital camera (Hamamatsu) controlled by Leica Application Suite X (Leica). Images were processed using Fiji $(98,99)$.

\section{Suppressor Screen}

Overnight M2X cultures of $\Delta c h v G$ and $\Delta c h v I$ cells were back-diluted to $\mathrm{OD}_{660}=0.025$ in $\mathrm{M} 2 \mathrm{X}$ medium. Cultures initially saturated at $\mathrm{OD}_{660} \sim 0.1$ before growing to higher density $\left(\mathrm{OD}_{660}=0.5-\right.$ 0.8 ) after 2-3 days growth. From each culture, single colonies were isolated on PYE plates. Isolated strains were then tested for suppression by M2X growth curves. The origin of each suppressor strain is as follows: culture $1(\Delta I S 1)$, culture $2(\Delta I S 2, \Delta I S 3)$, culture $3(\Delta G S 1)$, culture $4(\Delta G S 2$, $\triangle G S 3)$. Genomic DNA was isolated from $1 \mathrm{~mL}$ of overnight culture grown in PYE medium, using guanidinium thiocyanate (100). Sequencing was performed by the Microbial Genome Sequencing Center (Pittsburgh, PA) using a single library preparation method based upon the Illumina Nextera Kit. Sequences were aligned to the $C$. crescentus NA1000 reference genome (GenBank accession number CP001340) using breseq (101).

\section{Phos-tag gel electrophoresis and western blotting}

$2 \mathrm{~mL}$ M2X overnights of strains containing $n t r X-H A$ at the native $n t r X$ locus were grown and backdiluted to $\mathrm{OD}_{660}=0.01$. Samples were collected after $22 \mathrm{~h}\left(0.25 \mathrm{~mL} \cdot \mathrm{OD}_{660}\right.$; i.e. volume $[\mathrm{mL}]=$ $0.25 / \mathrm{OD}_{660}$ of culture) and frozen at $-80^{\circ} \mathrm{C}$. Samples were thawed, resuspended in $2.5 \mathrm{X}$ SDS loading buffer (125 mM Tris [pH 6.8], 25\% glycerol, 5\% SDS, $5 \mathrm{mM}$ DTT, 0.01\% bromophenol blue) containing 1:50 benzonase (Sigma), and immediately loaded onto Phos-tag gels.

Phos-tag electrophoresis was performed as described (48) using 8\% acrylamide gels copolymerized with $35 \mu \mathrm{M}$ Phos-tag acrylamide (NARD) and $150 \mu \mathrm{M} \mathrm{ZnCl}$. Proteins were transferred to polyvinylidene difluoride (PVDF; Bio-Rad) in a wet-transfer apparatus. Membranes were probed with monoclonal HA Tag antibody (1:2000 dilution, Invitrogen, 2-2.2.14,), incubated with anti-mouse IgG-horseradish peroxidase (IgG-HRP) conjugate (1:5000 dilution, Invitrogen), and developed with ProSignal ${ }^{\mathrm{TM}}$ Pico Spray (Prometheus). Blots were imaged using a Bio-Rad ChemiDoc MP Imager. Bands were quantified using Fiji $(98,99)$.

\section{Transcriptome Deep Sequencing (RNA-seq)}

$2 \mathrm{~mL}$ of PYE medium was inoculated with $\Delta c h v I \mathrm{EV}(\Delta c h v I x y l X:$ :pMT585) and $\Delta c h v I$ chvI(D52E)++ (AchvI xylX::pMT585-chvI(D52E)) cells and grown overnight. Cultures were diluted to $\mathrm{OD}_{660}=0.001$ in $2 \mathrm{~mL}$ fresh PYE and grown for $22.5 \mathrm{~h}$. Cultures were diluted to $\mathrm{OD}_{660}$ $=0.075$ in $5 \mathrm{~mL}$ PYE $+0.15 \%$ xylose and grown for $3.5 \mathrm{~h}$ before TRIzol extraction and RNA isolation, as described previously (102). RNA-seq libraries were prepared using an Illumina TruSeq stranded RNA kit and sequenced on an Illumina NextSEQ500 instrument at the University of Chicago Functional Genomics Facility. Sequencing data were analyzed using CLC Genomics 
Workbench 20 (Qiagen) by mapping reads to the C. crescentus NA1000 genome (84). Motif searching was carried out using MEME (103). Heatmaps were generated using Java Treeview3 and hypergeometric analysis was performed using phyper in $\mathrm{R}(104,105)$.

\section{$\beta$-galactosidase Assays}

For assays of WT vs. $\Delta c h v I$ strains, cells were grown overnight in $2 \mathrm{~mL}$ PYE medium. Then, 500 $\mu \mathrm{L}$ of each culture was centrifuged at $8 \mathrm{k} \times g$ for $3 \mathrm{~min}$ and resuspended in $500 \mu \mathrm{L}$ M2X medium. Resuspended cultures were used to inoculate $2 \mathrm{~mL} \mathrm{M2X}$ medium at a starting $\mathrm{OD}_{660}$ of 0.075. Cultures were grown for $4 \mathrm{~h}$ before assaying $\beta$-galactosidase activity. For assays of WT vs. $\Delta n t r X$ strains, cells were grown overnight in $2 \mathrm{~mL} \mathrm{M} 2 \mathrm{X}$ medium. Overnight cultures were diluted in $\mathrm{M} 2 \mathrm{X}$ medium such that they would reach $\mathrm{OD}_{660}=0.1-0.2$ after $23.5 \mathrm{~h}$ of growth. $\beta$-galactosidase assays were performed as previously described using $200 \mu \mathrm{L}$ of M2X culture $+100 \mu \mathrm{L}$ sterile PYE medium as an emulsifier (106). Plotting and statistical analyses were carried out using Prism (Graphpad).

\section{Data Availability}

RNA-seq data are available in the NCBI's Gene Expression Omnibus (GEO) database (107) under the GEP Series accession number (https://www.ncbi.nlm.nih.gov/geo/query/acc.cgi?acc=GSE168965).

\section{Acknowledgements}

We thank Clare Kirkpatrick, Régis Hallez, Alex Quintero, and members of the Crosson laboratory for helpful discussion, and Jen Mach and Plant Editors for constructive feedback on the manuscript. Research in this publication was supported by the National Institute of General Medical Sciences of the National Institutes of Health (NIH) under Award Numbers F32 GM128283 (B.J.S.) and R35 GM131762 (S.C.). The content is solely the responsibility of the authors and does not necessarily represent the official views of the National Institutes of Health. 


\section{References}

1. Stock AM, Robinson VL, Goudreau PN. 2000. Two-Component Signal Transduction. Annu Rev Biochem 69:183-215.

2. Crosson S, McGrath PT, Stephens C, McAdams HH, Shapiro L. 2005. Conserved modular design of an oxygen sensory/signaling network with species-specific output. Proc Natl Acad Sci U S A 102:8018-8023.

3. David M, Daveran ML, Batut J, Dedieu A, Domergue O, Ghai J, Hertig C, Boistard P, Kahn D. 1988. Cascade Regulation of nif Gene Expression in Rhizobium meliloti. Cell 54:671683.

4. Viadas C, Rodríguez MC, Sangari FJ, Gorvel JP, García-Lobo JM, López-Goñi I. 2010. Transcriptome Analysis of the Brucella abortus BvrR/BvrS Two-Component Regulatory System. PLoS ONE 5:e10216.

5. Ratib NR, Sabio EY, Mendoza C, Barnett MJ, Clover SB, Ortega JA, Cruz Dela FM, Balderas D, White H, Long SR, Chen EJ. 2018. Genome-wide identification of genes directly regulated by ChvI and a consensus sequence for ChvI binding in Sinorhizobium meliloti. Mol Microbiol 110:596-615.

6. Fröhlich KS, Förstner KU, Gitai Z. 2018. Post-transcriptional gene regulation by an Hfqindependent small RNA in Caulobacter crescentus. Nucleic Acids Res 66:325.

7. Landa AS, Cerdá JP, Grilló MJ, Moreno E, Moriyón I, Blasco JM, Gorvel JP, Goñi IL. 1998. A two-component regulatory system playing a critical role in plant pathogens and endosymbionts is present in Brucella abortus and controls cell invasion and virulence. Mol Microbiol 29:125-138.

8. Charles TC, Nester EW. 1993. A chromosomally encoded two-component sensory transduction system is required for virulence of Agrobacterium tumefaciens. J Bacteriol 175:6614-6625.

9. Gottschlich L, Geiser P, Bortfeld-Miller M, Field CM, Vorholt JA. 2019. Complex general stress response regulation in Sphingomonas melonis Fr1 revealed by transcriptional analyses. Sci Rep 9:9404.

10. Kaczmarczyk A, Hochstrasser R, Vorholt JA, Francez-Charlot A. 2014. Complex twocomponent signaling regulates the general stress response in Alphaproteobacteria. Proc Natl Acad Sci U S A 111:E5196-204.

11. Lori C, Kaczmarczyk A, de Jong I, Jenal U. 2018. A Single-Domain Response Regulator Functions as an Integrating Hub To Coordinate General Stress Response and Development in Alphaproteobacteria. mBio 9:e00809-18.

12. Francis VI, Porter SL. 2019. Multikinase Networks: Two-Component Signaling Networks Integrating Multiple Stimuli. Annu Rev Microbiol 73:199-223. 
13. Howell A, Dubrac S, Noone D, Varughese KI, Devine K. 2006. Interactions between the YycFG and PhoPR two-component systems in Bacillus subtilis: the PhoR kinase phosphorylates the non-cognate $\mathrm{YycF}$ response regulator upon phosphate limitation. Mol Microbiol 59:1199-1215.

14. Perego M, Hanstein C, Welsh KM, Djavakhishvili T, Glaser P, Hoch JA. 1994. Multiple Protein-Aspartate Phosphatases Provide a Mechanism for the Integration of Diverse Signals in the Control of Development in B. subtilis. Cell 79:1047-1055.

15. Wells DH, Chen EJ, Fisher RF, Long SR. 2007. ExoR is genetically coupled to the ExoSChvi two-component system and located in the periplasm of Sinorhizobium meliloti. Mol Microbiol 64:647-664.

16. Lu H-Y, Luo L, Yang M-H, Cheng H-P. 2012. Sinorhizobium meliloti ExoR Is the Target of Periplasmic Proteolysis. J Bacteriol 194:4029-4040.

17. Stein BJ, Fiebig A, Crosson S. 2020. Feedback Control of a Two-Component Signaling System by an Fe-S-Binding Receiver Domain. mBio 11:e03383-19.

18. Noriega CE, Lin H-Y, Chen L-L, Williams SB, Stewart V. 2010. Asymmetric crossregulation between the nitrate-responsive NarX-NarL and NarQ-NarP two-component regulatory systems from Escherichia coli K-12. Mol Microbiol 75:394-412.

19. Miller MB, Skorupski K, Lenz DH, Taylor RK, Bassler BL. 2002. Parallel quorum sensing systems converge to regulate virulence in Vibrio cholerae. Cell 110:303-314.

20. Skerker JM, Prasol MS, Perchuk BS, Biondi EG, Laub MT. 2005. Two-Component Signal Transduction Pathways Regulating Growth and Cell Cycle Progression in a Bacterium: A System-Level Analysis. PLoS Biol 3:e334.

21. Bijlsma JJE, Groisman EA. 2003. Making informed decisions: regulatory interactions between two-component systems. Trends Microbiol 11:359-366.

22. Birkey SM, Liu W, Zhang X, Duggan MF, Hulett FM. 1998. Pho signal transduction network reveals direct transcriptional regulation of one two-component system by another two-component regulator: Bacillus subtilis PhoP directly regulates production of ResD. Mol Microbiol 30:943-953.

23. Mouslim C, Groisman EA. 2003. Control of the Salmonella ugd gene by three twocomponent regulatory systems. Mol Microbiol 47:335-344.

24. Huang J, Carney BF, Denny TP, Weissinger AK, Schell MA. 1995. A complex network regulates expression of eps and other virulence genes of Pseudomonas solanacearum. J Bacteriol 177:1259-1267.

25. Wang D, Xue H, Wang Y, Yin R, Xie F, Luo L. 2013. The Sinorhizobium meliloti ntrX Gene Is Involved in Succinoglycan Production, Motility, and Symbiotic Nodulation on Alfalfa. Appl Environ Microbiol 79:7150-7159. 
26. Heckel BC, Tomlinson AD, Morton ER, Choi JH, Fuqua C. 2014. Agrobacterium tumefaciens ExoR Controls Acid Response Genes and Impacts Exopolysaccharide Synthesis, Horizontal Gene Transfer, and Virulence Gene Expression. J Bacteriol 196:3221-3233.

27. Mantis NJ, Winans SC. 1993. The chromosomal response regulatory gene chvI of Agrobacterium tumefaciens complements an Escherichia coli phoB mutation and is required for virulence. J Bacteriol 175:6626-6636.

28. Cheng HP, Walker GC. 1998. Succinoglycan production by Rhizobium meliloti is regulated through the ExoS-ChvI two-component regulatory system. J Bacteriol 180:20-26.

29. Guzman-Verri C, Manterola L, Sola-Landa A, Parra A, Cloeckaert A, Garin J, Gorvel JP, Moriyon I, Moreno E, Lopez-Goni I. 2002. The two-component system BvrR/BvrS essential for Brucella abortus virulence regulates the expression of outer membrane proteins with counterparts in members of the Rhizobiaceae. Proc Natl Acad Sci U S A 99:1237512380 .

30. Yao SY, Luo L, Har KJ, Becker A, Ruberg S, Yu GQ, Zhu JB, Cheng HP. 2004. Sinorhizobium meliloti ExoR and ExoS Proteins Regulate both Succinoglycan and Flagellum Production. J Bacteriol 186:6042-6049.

31. Foreman DL, Vanderlinde EM, Bay DC, Yost CK. 2010. Characterization of a Gene Family of Outer Membrane Proteins (ropB) in Rhizobium leguminosarum bv. viciae VF39SM and the Role of the Sensor Kinase ChvG in Their Regulation. J Bacteriol 192:975-983.

32. Quebatte M, Dehio M, Tropel D, Basler A, Toller I, Raddatz G, Engel P, Huser S, Schein H, Lindroos HL, Andersson SGE, Dehio C. 2010. The BatR/BatS Two-Component Regulatory System Controls the Adaptive Response of Bartonella henselae during Human Endothelial Cell Infection. J Bacteriol 192:3352-3367.

33. Chen EJ, Sabio EA, Long SR. 2008. The periplasmic regulator ExoR inhibits ExoS/ChvI two-component signalling in Sinorhizobium meliloti. Mol Microbiol 69:1290-1303.

34. Wu C-F, Lin J-S, Shaw G-C, Lai E-M. 2012. Acid-Induced Type VI Secretion System Is Regulated by ExoR-ChvG/ChvI Signaling Cascade in Agrobacterium tumefaciens. PLOS Pathog 8:e1002938.

35. Pawlowski K, Klosse U, de Bruijn FJ. 1991. Characterization of a novel Azorhizobium caulinodans ORS571 two-component regulatory system, NtrY/NtrX, involved in nitrogen fixation and metabolism. Molec Gen Genet 231:124-138.

36. Ishida ML, Assumpção MC, Machado HB, Benelli EM, Souza EM, Pedrosa FO. 2002. Identification and characterization of the two-component $\mathrm{NtrY} / \mathrm{NtrX}$ regulatory system in Azospirillum brasilense. Braz J Med Biol Res 35:651-661. 
37. Cheng Z, Lin M, Rikihisa Y. 2014. Ehrlichia chaffeensis Proliferation Begins with NtrY/NtrX and PutA/GlnA Upregulation and CtrA Degradation Induced by Proline and Glutamine Uptake. mBio 5:e02141-14.

38. Atack JM, Srikhanta YN, Djoko KY, Welch JP, Hasri NHM, Steichen CT, Hoven RNV, Grimmond SM, Othman DSMP, Kappler U, Apicella MA, Jennings MP, Edwards JL, McEwan AG. 2013. Characterization of an $n t r X$ Mutant of Neisseria gonorrhoeae Reveals a Response Regulator That Controls Expression of Respiratory Enzymes in OxidasePositive Proteobacteria. J Bacteriol 195:2632-2641.

39. del Carmen Carrica M, Fernández I, Martí MA, Paris G, Goldbaum FA. 2012. The NtrY/X two-component system of Brucella spp. acts as a redox sensor and regulates the expression of nitrogen respiration enzymes. Mol Microbiol 85:39-50.

40. Lemmer KC, Alberge F, Myers KS, Dohnalkova AC, Schaub RE, Lenz JD, Imam S, Dillard JP, Noguera DR, Donohue TJ. 2020. The NtrYX Two-Component System Regulates the Bacterial Cell Envelope. mBio 11:e00957-20.

41. Calatrava-Morales N, Nogales J, Ameztoy K, van Steenbergen B, Soto MJ. 2017. The NtrY/NtrX System of Sinorhizobium meliloti GR4 Regulates Motility, EPS I Production, and Nitrogen Metabolism but Is Dispensable for Symbiotic Nitrogen Fixation. MPMI 30:566-577.

42. Bonato P, Alves LR, Osaki JH, Rigo LU, Pedrosa FO, Souza EM, Zhang N, Schumacher J, Buck M, Wassem R, Chubatsu LS. 2016. The NtrY-NtrX two-component system is involved in controlling nitrate assimilation in Herbaspirillum seropedicae strain SmR1. FEBS J 283:3919-3930.

43. Urtecho G, Campbell DE, Hershey DM, Hussain FA, Whitaker RJ, O'Toole GA. 2020. Discovering the Molecular Determinants of Phaeobacter inhibens Susceptibility to Phaeobacter Phage MD18. mSphere 5:666.

44. Drepper T, Wiethaus J, Giaourakis D, GroÃ S, Schubert B, Vogt M, Wiencek Y, McEwan AG, Masepohl B. 2006. Cross-talk towards the response regulator NtrC controlling nitrogen metabolism in Rhodobacter capsulatus. FEMS Microbiol Lett 258:250-256.

45. Poindexter JS. 1964. Biological properties and classification of the Caulobacter group. Bacteriol Rev 28:231-295.

46. Wilhelm RC. 2018. Following the terrestrial tracks of Caulobacter - redefining the ecology of a reputed aquatic oligotroph. ISME J 12:3025-3037.

47. Vallet S-U, Hansen LH, Bistrup FC, Laursen SA, Chapalay JB, Chambon M, Turcatti G, Viollier PH, Kirkpatrick CL. 2020. Loss of Bacterial Cell Pole Stabilization in Caulobacter crescentus Sensitizes to Outer Membrane Stress and Peptidoglycan-Directed Antibiotics. mBio 11:e00538-20. 
48. Fernández I, Sycz G, Goldbaum FA, del Carmen Carrica M. 2018. Acidic pH triggers the phosphorylation of the response regulator $\mathrm{NtrX}$ in alphaproteobacteria. PLoS ONE 13:e0194486.

49. Capra EJ, Perchuk BS, Skerker JM, Laub MT. 2012. Adaptive Mutations that Prevent Crosstalk Enable the Expansion of Paralogous Signaling Protein Families. Cell 150:222232.

50. Karl KE, David WS, Sydney K. 1993. Glutamate at the site of phosphorylation of nitrogenregulatory protein NTRC mimics aspartyl-phosphate and activates the protein. J Mol Bio 232:67-78.

51. Moore JB, Shiau SP, Reitzer LJ. 1993. Alterations of highly conserved residues in the regulatory domain of nitrogen regulator I (NtrC) of Escherichia coli. J Bacteriol 175:26922701.

52. Gupte G, Woodward C, Stout V. 1997. Isolation and characterization of $\operatorname{rcs} B$ mutations that affect colanic acid capsule synthesis in Escherichia coli K-12. J Bacteriol 179:4328-4335.

53. Lan CY, Igo MM. 1998. Differential expression of the OmpF and OmpC porin proteins in Escherichia coli K-12 depends upon the level of active OmpR. J Bacteriol 180:171-174.

54. Gushchin I, Gordeliy V. 2018. Transmembrane Signal Transduction in Two-Component Systems: Piston, Scissoring, or Helical Rotation? Bioessays 40:1700197.

55. Gushchin I, Melnikov I, Polovinkin V, Ishchenko A, Yuzhakova A, Buslaev P, Bourenkov G, Grudinin S, Round E, Balandin T, Borshchevskiy V, Willbold D, Leonard G, Büldt G, Popov A, Gordeliy V. 2017. Mechanism of transmembrane signaling by sensor histidine kinases. Science 356:eaah6345.

56. Molnar KS, Bonomi M, Pellarin R, Clinthorne GD, Gonzalez G, Goldberg SD, Goulian M, Sali A, DeGrado WF. 2014. Cys-Scanning Disulfide Crosslinking and Bayesian Modeling Probe the Transmembrane Signaling Mechanism of the Histidine Kinase, PhoQ. Structure 22:1239-1251.

57. Skerker JM, Perchuk BS, Siryaporn A, Lubin EA, Ashenberg O, Goulian M, Laub MT. 2008. Rewiring the Specificity of Two-Component Signal Transduction Systems. Cell 133:1043-1054.

58. Yamada S, Sugimoto H, Kobayashi M, Ohno A, Nakamura H, Shiro Y. 2009. Structure of PAS-Linked Histidine Kinase and the Response Regulator Complex. Structure 17:13331344.

59. Bélanger L, Dimmick KA, Fleming JS, Charles TC. 2009. Null mutations in Sinorhizobium meliloti exoS and chvI demonstrate the importance of this two-component regulatory system for symbiosis. Mol Microbiol 74:1223-1237. 
60. Lourenço RF, Kohler C, Gomes SL. 2011. A two-component system, an anti-sigma factor and two paralogous ECF sigma factors are involved in the control of general stress response in Caulobacter crescentus. Mol Microbiol 80:1598-1612.

61. Alvarez-Martinez CE, Lourenço RF, Baldini RL, Laub MT, Gomes SL. 2007. The ECF sigma factor $\sigma$ Tis involved in osmotic and oxidative stress responses in Caulobacter crescentus. Mol Microbiol 66:1240-1255.

62. Alvarez-Martinez CE, Baldini RL, Gomes SL. 2006. A Caulobacter crescentus Extracytoplasmic Function Sigma Factor Mediating the Response to Oxidative Stress in Stationary Phase. J Bacteriol 188:1835-1846.

63. Hentchel KL, Ruiz LMR, Curtis PD, Fiebig A, Coleman ML, Crosson S. 2019. Genomescale fitness profile of Caulobacter crescentus grown in natural freshwater. ISME J 13:523536.

64. Chen EJ, Fisher RF, Perovich VM, Sabio EA, Long SR. 2009. Identification of Direct Transcriptional Target Genes of ExoS/ChvI Two-Component Signaling in Sinorhizobium meliloti. J Bacteriol 191:6833-6842.

65. Herrou J, Willett JW, Fiebig A, Varesio LM, Czyż DM, Cheng JX, Ultee E, Briegel A, Bigelow L, Babnigg G, Kim Y, Crosson S. 2019. Periplasmic protein EipA determines envelope stress resistance and virulence in Brucella abortus. Mol Microbiol 111:637-661.

66. Hagan CL, Silhavy TJ, Kahne D. 2011. $\beta$-Barrel Membrane Protein Assembly by the Bam Complex. Annu Rev Biochem 80:189-210.

67. Anwari K, Poggio S, Perry A, Gatsos X, Ramarathinam SH, Williamson NA, Noinaj N, Buchanan S, Gabriel K, Purcell AW, Jacobs-Wagner C, Lithgow T. 2010. A modular BAM complex in the outer membrane of the alpha-proteobacterium Caulobacter crescentus. PLoS ONE 5:e8619.

68. Yeh Y-C, Comolli LR, Downing KH, Shapiro L, McAdams HH. 2010. The Caulobacter Tol-Pal complex is essential for outer membrane integrity and the positioning of a polar localization factor. J Bacteriol 192:4847-4858.

69. Wang C, Kemp J, Da Fonseca IO, Equi RC, Sheng X, Charles TC, Sobral BWS. 2010. Sinorhizobium meliloti 1021 Loss-of-Function Deletion Mutation in chvI and Its Phenotypic Characteristics. MPMI 23:153-160.

70. Tomlinson AD, Ramey-Hartung B, Day TW, Merritt PM, Fuqua C. 2010. Agrobacterium tumefaciens ExoR represses succinoglycan biosynthesis and is required for biofilm formation and motility. Microbiology 156:2670-2681.

71. Aldridge P, Jenal U. 1999. Cell cycle-dependent degradation of a flagellar motor component requires a novel-type response regulator. Mol Microbiol 32:379-391. 
72. Hershey DM, Fiebig A, Crosson S. 2021. Flagellar Perturbations Activate Adhesion through Two Distinct Pathways in Caulobacter crescentus. mBio 12:e03266-20.

73. Ardissone S, Viollier PH. 2015. Interplay between flagellation and cell cycle control in Caulobacter. Curr Opin Microbiol 28:83-92.

74. Woldemeskel SA, McQuillen R, Hessel AM, Xiao J, Goley ED. 2017. A conserved coiledcoil protein pair focuses the cytokinetic Z-ring in Caulobacter crescentus. Mol Microbiol 105:721-740.

75. Schwartz MA, Shapiro L. 2011. An SMC ATPase mutant disrupts chromosome segregation in Caulobacter. Mol Microbiol 82:1359-1374.

76. Jensen RB, Shapiro L. 1999. The Caulobacter crescentus smc gene is required for cell cycle progression and chromosome segregation. Proc Natl Acad Sci U S A 96:10661-10666.

77. Narayanan S, Janakiraman B, Kumar L, Radhakrishnan SK. 2015. A cell cycle-controlled redox switch regulates the topoisomerase IV activity. Genes Dev 29:1175-1187.

78. Schindel HS, Bauer CE. 2016. The RegA regulon exhibits variability in response to altered growth conditions and differs markedly between Rhodobacter species. Microb Genom 2:e000081.

79. Feldheim YS, Zusman T, Speiser Y, Segal G. 2016. The Legionella pneumophila CpxRA two-component regulatory system: new insights into CpxR's function as a dual regulator and its connection to the effectors regulatory network. Mol Microbiol 99:1059-1079.

80. Plate L, Marletta MA. 2013. Phosphorylation-dependent derepression by the response regulator HnoC in the Shewanella oneidensis nitric oxide signaling network. Proc Natl Acad Sci U S A 110:E4648-57.

81. Chakraborty S, Winardhi RS, Morgan LK, Yan J, Kenney LJ. 2017. Non-canonical activation of OmpR drives acid and osmotic stress responses in single bacterial cells. Nat Comms 8:1587.

82. Ali MM, Provoost A, Mijnendonckx K, Van Houdt R, Charlier D. 2020. DNA-Binding and Transcription Activation by Unphosphorylated Response Regulator AgrR From Cupriavidus metallidurans Involved in Silver Resistance. Front Microbiol 11:1635.

83. Fernández I, Cornaciu I, Carrica MDC, Uchikawa E, Hoffmann G, Sieira R, Márquez JA, Goldbaum FA. 2017. Three-Dimensional Structure of Full-Length NtrX, an Unusual Member of the NtrC Family of Response Regulators. J Mol Bio 429:1192-1212.

84. Marks ME, Castro-Rojas CM, Teiling C, Du L, Kapatral V, Walunas TL, Crosson S. 2010. The Genetic Basis of Laboratory Adaptation in Caulobacter crescentus. J Bacteriol 192:3678-3688. 
85. García-Bayona L, Gozzi K, Laub MT. 2019. Mechanisms of Resistance to the ContactDependent Bacteriocin CdzC/D in Caulobacter crescentus. J Bacteriol 201:e00538-18.

86. Hu X, Zhao J, DeGrado WF, Binns AN. 2013. Agrobacterium tumefaciens recognizes its host environment using ChvE to bind diverse plant sugars as virulence signals. Proc Natl Acad Sci U S A 110:678-683.

87. Göpel Y, Görke B. 2018. Interaction of lipoprotein QseG with sensor kinase QseE in the periplasm controls the phosphorylation state of the two-component system QseE/QseF in Escherichia coli. PLOS Genet 14:e1007547.

88. Lippa AM, Goulian M. 2009. Feedback Inhibition in the PhoQ/PhoP Signaling System by a Membrane Peptide. PLOS Genet 5:e1000788.

89. Lukat GS, McCleary WR, Stock AM, Stock JB. 1992. Phosphorylation of bacterial response regulator proteins by low molecular weight phospho-donors. Proc Natl Acad Sci U S A 89:718-722.

90. McCleary WR, Stock JB. 1994. Acetyl phosphate and the activation of two-component response regulators. J Biol Chem 269:31567-31572.

91. Kim S-B, Shin B-S, Choi S-K, Kim C-K, Park S-H. 2001. Involvement of acetyl phosphate in the in vivo activation of the response regulator ComA in Bacillus subtilis. FEMS Microbiol Lett 195:179-183.

92. Bouché S, Klauck E, Fischer D, Lucassen M, Jung K, Hengge-Aronis R. 1998. Regulation of RssB-dependent proteolysis in Escherichia coli: a role for acetyl phosphate in a response regulator-controlled process. Mol Microbiol 27:787-795.

93. Hsing W, Russo FD, Bernd KK, Silhavy TJ. 1998. Mutations that alter the kinase and phosphatase activities of the two-component sensor EnvZ. J Bacteriol 180:4538-4546.

94. Lemmin T, Soto CS, Clinthorne G, DeGrado WF, Peraro MD. 2013. Assembly of the Transmembrane Domain of E. coli PhoQ Histidine Kinase: Implications for Signal Transduction from Molecular Simulations. PLOS Comput Biol 9:e1002878.

95. Goldberg SD, Clinthorne GD, Goulian M, DeGrado WF. 2010. Transmembrane polar interactions are required for signaling in the Escherichia coli sensor kinase PhoQ. Proc Natl Acad Sci U S A 107:8141-8146.

96. Wiech EM, Cheng H-P, Singh SM. 2015. Molecular modeling and computational analyses suggests that the Sinorhizobium meliloti periplasmic regulator protein ExoR adopts a superhelical fold and is controlled by a unique mechanism of proteolysis. Protein Sci 24:319-327.

97. Ried JL, Collmer A. 1987. An $n p t I-s a c B$-sacR cartridge for constructing directed, unmarked mutations in Gram-negative bacteria by marker exchange-eviction mutagenesis. Gene 57:239-246. 
98. Schindelin J, Arganda-Carreras I, Frise E, Kaynig V, Longair M, Pietzsch T, Preibisch S, Rueden C, Saalfeld S, Schmid B, Tinevez J-Y, White DJ, Hartenstein V, Eliceiri K, Tomancak P, Cardona A. 2012. Fiji: an open-source platform for biological-image analysis. Nat Meth 9:676-682.

99. Schneider CA, Rasband WS, Eliceiri KW. 2012. NIH Image to ImageJ: 25 years of image analysis. Nat Meth 9:671-675.

100. Pitcher DG, Saunders NA, Owen RJ. 1989. Rapid extraction of bacterial genomic DNA with guanidium thiocyanate. Lett Appl Microbiol 8:151-156.

101. Deatherage DE, Barrick JE. 2014. Identification of mutations in laboratory evolved microbes from next-generation sequencing data using breseq. Methods Mol Biol 1151:165188.

102. Tien MZ, Stein BJ, Crosson S. 2018. Coherent Feedforward Regulation of Gene Expression by Caulobacter $\sigma \mathrm{T}$ and GsrN during Hyperosmotic Stress. J Bacteriol 200:e00349-18.

103. Bailey TL, Boden M, Buske FA, Frith M, Grant CE, Clementi L, Ren J, Li WW, Noble WS. 2009. MEME SUITE: tools for motif discovery and searching. Nucleic Acids Res 37:W202-W208.

104. Saldanha AJ. 2004. Java Treeview-extensible visualization of microarray data. Bioinformatics 20:3246-3248.

105. R Core Team. 2020. R: A language and environment for statistical computing. R Foundation for Statistical Computing, Vienna, Austria. https://wwwR-project.org/

106. Foreman R, Fiebig A, Crosson S. 2012. The LovK-LovR Two-Component System Is a Regulator of the General Stress Pathway in Caulobacter crescentus. J Bacteriol 194:30383049 .

107. Edgar R, Domrachev M, Lash AE. 2002. Gene Expression Omnibus: NCBI gene expression and hybridization array data repository. Nucleic Acids Res. 30(1):207-10. https://www.ncbi.nlm.nih.gov/geo/ 